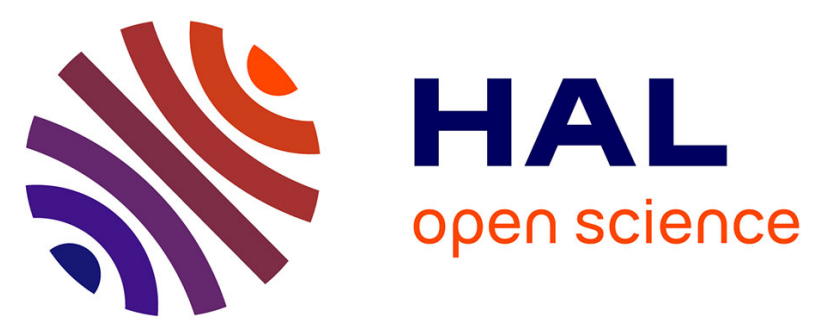

\title{
Comparative Analysis of the Various Generalized Ohm's Law Terms in Magnetosheath Turbulence as Observed by Magnetospheric Multiscale
}

J. Stawarz, L. Matteini, T. Parashar, L. Franci, J. Eastwood, C. A Gonzalez, I. L Gingell, J. Burch, R. Ergun, N. Ahmadi, et al.

\section{To cite this version:}

J. Stawarz, L. Matteini, T. Parashar, L. Franci, J. Eastwood, et al.. Comparative Analysis of the Various Generalized Ohm's Law Terms in Magnetosheath Turbulence as Observed by Magnetospheric Multiscale. Journal of Geophysical Research Space Physics, 2021, 126 (1), 10.1029/2020ja028447. hal-03193745

\section{HAL Id: hal-03193745 \\ https://hal.sorbonne-universite.fr/hal-03193745}

Submitted on 9 Apr 2021

HAL is a multi-disciplinary open access archive for the deposit and dissemination of scientific research documents, whether they are published or not. The documents may come from teaching and research institutions in France or abroad, or from public or private research centers.
L'archive ouverte pluridisciplinaire HAL, est destinée au dépôt et à la diffusion de documents scientifiques de niveau recherche, publiés ou non, émanant des établissements d'enseignement et de recherche français ou étrangers, des laboratoires publics ou privés. 


\section{JGR Space Physics}

\section{RESEARCH ARTICLE \\ 10.1029/2020JA028447 \\ Key Points: \\ - The role of generalized Ohm's law in shaping the turbulent electric field spectrum is examined from magnetohydrodynamic to electron scales \\ - The electron pressure term is more significant than expected from linear kinetic Alfvén waves and partially antialigns with the Hall term \\ - Relative contributions of linear and nonlinear electric fields are constant with scale and given by average turbulent fluctuation amplitude}

Correspondence to:

J. E. Stawarz,

j.stawarz@imperial.ac.uk

Citation:

Stawarz, J. E., Matteini, L., Parashar, T. N., Franci, L., Eastwood, J. P., Gonzalez, C. A., et al. (2021).

Comparative analysis of the various generalized Ohm's law terms in magnetosheath turbulence as observed by Magnetospheric Multiscale. Journal of Geophysical Research: Space Physics, 126, e2020JA028447. https://doi. org/10.1029/2020JA028447

Received 4 JUL 2020 Accepted 13 NOV 2020 (c) 2020. The Authors.

This is an open access article under the terms of the Creative Commons Attribution License, which permits use, distribution and reproduction in any medium, provided the original work is properly cited.
Comparative Analysis of the Various Generalized Ohm's Law Terms in Magnetosheath Turbulence as Observed by Magnetospheric Multiscale

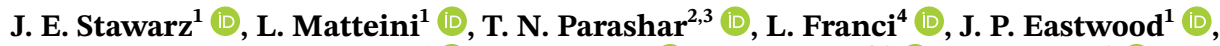 \\ C. A. Gonzalez ${ }^{5}$, I. L. Gingell ${ }^{6}$ (D), J. L. Burch ${ }^{7}$ iD, R. E. Ergun ${ }^{8,9}$ (D) N. Ahmadi ${ }^{9}$ (iD, \\ B. L. Giles ${ }^{10}$ (D) D. J. Gershman ${ }^{10}$ (D), O. Le Contel ${ }^{11}$ (D) P.-A. Lindqvist ${ }^{12}$ (D) C. T. Russell ${ }^{13}$ (D), \\ R. J. Strangeway ${ }^{13}$, and R. B. Torbert ${ }^{14}$ iD \\ ${ }^{1}$ Department of Physics, Imperial College London, London, UK, ${ }^{2}$ School of Chemical and Physical Sciences, \\ Victoria University of Wellington, Wellington, New Zealand, ${ }^{3}$ Department of Physics and Astronomy, University of \\ Delaware, Newark, DE, USA, ${ }^{4}$ Department of Physics and Astronomy, Queen Mary University of London, London, \\ UK, ${ }^{5}$ Department of Physics, University of Texas at Austin, Austin, TX, USA, ${ }^{6}$ School of Physics and Astronomy, \\ University of Southampton, Southampton, UK, ${ }^{7}$ Southwest Research Institute, San Antonio, TX, USA, ${ }^{8}$ Department \\ of Astrophysical and Planetary Sciences, University of Colorado, Boulder, CO, USA, ${ }^{9}$ Laboratory for Atmospheric and \\ Space Physics, University of Colorado, Boulder, CO, USA, ${ }^{10}$ NASA Goddard Space Flight Center, Greenbelt, MD, USA, \\ ${ }^{11}$ Laboratoire de Physique des Plasmas, CNRS, Ecole Polytechnique, Sorbonne Université, Université Paris-Saclay, \\ Observatoire de Paris, Paris, France, ${ }^{12}$ School of Electrical Engineering, KTH Royal Institute of Technology, Stockholm, \\ Sweden, ${ }^{13}$ Department of Earth, Planetary, and Space Sciences, University of California, Los Angeles, CA, USA, \\ ${ }^{14}$ Department of Physics, University of New Hampshire, Durham, NH, USA
}

Abstract Decomposing the electric field (E) into the contributions from generalized Ohm's law provides key insight into both nonlinear and dissipative dynamics across the full range of scales within a plasma. Using high-resolution, multispacecraft measurements of three intervals in Earth's magnetosheath from the Magnetospheric Multiscale mission, the influence of the magnetohydrodynamic, Hall, electron pressure, and electron inertia terms from Ohm's law, as well as the impact of a finite electron mass, on the turbulent $\mathbf{E}$ spectrum are examined observationally for the first time. The magnetohydrodynamic, Hall, and electron pressure terms are the dominant contributions to $\mathbf{E}$ over the accessible length scales, which extend to scales smaller than the electron gyroradius at the greatest extent, with the Hall and electron pressure terms dominating at sub-ion scales. The strength of the nonideal electron pressure contribution is stronger than expected from linear kinetic Alfvén waves and a partial antialignment with the Hall electric field is present, linked to the relative importance of electron diamagnetic currents in the turbulence. The relative contribution of linear and nonlinear electric fields scale with the turbulent fluctuation amplitude, with nonlinear contributions playing the dominant role in shaping $\mathbf{E}$ for the intervals examined in this study. Overall, the sum of the Ohm's law terms and measured $\mathbf{E}$ agree to within $\sim 20 \%$ across the observable scales. These results both confirm general expectations about the behavior of $\mathbf{E}$ in turbulent plasmas and highlight features that should be explored further theoretically.

Plain Language Summary Complex turbulent motions are observed in plasmas throughout the Universe and act to transfer energy from large-scale fluctuations to small-scale fluctuations, which can be more easily dissipated into the thermal energy of the particles. Electric fields in these plasmas play a central role in enabling the exchange of energy between the magnetic field and the motion of the charged particles and are, therefore, important for disentangling the complex nonlinear dynamics and dissipative processes. Using cutting-edge, high-resolution, multispacecraft measurements from NASA's Magnetospheric Multiscale mission, we decompose the electric field in Earth's turbulent magnetosheath into the various terms from generalized Ohm's law, which governs the behavior of the electric field across the wide range of length scales in the plasma. The results confirm a number of general expectations about the relative behavior of the terms in Ohm's law, as well as highlight several new features that are significant for understanding the nonlinear behavior and turbulent dissipation at different scales within the plasma. 


\section{Introduction}

Turbulent dynamics are characterized by nonlinear interactions that transfer energy between fluctuations at different length scales within a fluid, often from large to small scales, generating multiscale gradients and facilitating the dissipation of the fluctuations. Many plasmas are either directly observed or thought to be turbulent, including the solar corona (e.g., Cranmer et al., 2015), solar wind (e.g., Bruno \& Carbone, 2013), planetary magnetospheres (e.g., Borovsky et al., 1997; Sahraoui et al., 2004; Saur et al., 2002; Stawarz et al., 2016), interstellar medium (e.g., Falceta-Gonçalves et al., 2014), accretion discs (e.g., Kawazura et al., 2019), and intracluster medium (e.g., Zhuravleva et al., 2014), and turbulent dissipation contributes to particle acceleration and heating within these systems. Fluctuations within turbulent plasmas can take the form of nonlinearly interacting normal modes (Boldyrev et al., 2013; Chen \& Boldyrev, 2017), which have different characteristics across the various length scales, as well as nonlinear structures, such as current sheets that can be sites for magnetic reconnection (Chasapis, Matthaeus, et al., 2018; Phan et al., 2018; Stawarz et al., 2019).

A significant amount of the fluctuation energy within a turbulent plasma can be carried by the magnetic field (B) (Gershman et al., 2018; Matthaeus \& Goldstein, 1982). However, since B cannot do work on the plasma, energy exchange between the electromagnetic fields and particles is mediated by the electric field (E) through a nonzero $\mathbf{j} \cdot \mathbf{E}$, where $\mathbf{j}$ is the electric current density. The energy transfer between the fields and particles both enables many of the nonlinear couplings that give rise to turbulence and provides pathways for the dissipation of electromagnetic fluctuations (Chasapis, Yang, et al., 2018; Chen et al., 2019; Ergun et al., 2018).

Within a collisionless plasma, $\mathbf{E}$ is governed by generalized Ohm's law, which arises from the electron and/ or ion fluid momentum equations and can be expressed in terms of $\mathbf{j}$ and the single-fluid velocity (u) for a two species plasma as (e.g., Baumjohann \& Treumann, 1996)

$$
\mathbf{E}=-\mathbf{u} \times \mathbf{B}+\frac{1}{e n} \mathbf{j} \times \mathbf{B}-\frac{1}{e n} \nabla \cdot \mathbf{p}_{e}+\frac{m_{e}}{e^{2} n}\left[\nabla \cdot\left(\mathbf{u j}+\mathbf{j u}-\frac{\mathbf{j} \mathbf{j}}{e n}\right)+\frac{\partial \mathbf{j}}{\partial t}\right]+\sum_{\infty}^{\ell=1}\left(-\frac{m_{e}}{m_{i}}\right)^{\ell} \mathcal{M}_{\ell},
$$

where $e$ is the elementary charge, $m_{i}$ and $m_{e}$ are the ion and electron masses, $n$ is the ion or electron number density, which are taken to be equal due to quasineutrality, $\mathbf{p}_{e}$ is the electron pressure tensor, and $\mathbf{u j}, \mathbf{j u}$, and $\mathbf{j j}$ denote the outer product of the two vectors. $\mathcal{M}_{\ell}$ denotes the $\ell^{\text {th }}$-order, finite-electron-mass corrections to the single-fluid formulation, resulting from Taylor expanding about small $m_{e} / m_{i}$ and is given by

$$
\mathcal{M}_{\ell}=\frac{2}{e n} \mathbf{j} \times \mathbf{B}-\frac{1}{e n} \nabla \cdot\left(\mathbf{p}_{e}+\mathbf{p}_{i}\right)+\frac{m_{e}}{e^{2} n}\left[\nabla \cdot\left(\mathbf{u j}+\mathbf{j u}-(1+2 \ell) \frac{\mathbf{j} \mathbf{j}}{e n}\right)+\frac{\partial \mathbf{j}}{\partial t}\right]
$$

with $\mathbf{p}_{i}$ the ion pressure tensor. The electric field associated with collisional resistivity is omitted in the above expressions, since collisions are expected to be rare within the magnetosheath.

When expressed in single-fluid variables, the terms in Ohm's law highlight the underlying dynamics operating in the plasma across the magnetohydrodynamic (MHD), sub-ion, and electron scales. The first term on the right-hand-side (r.h.s.) of Equation $1\left(\mathbf{E}_{M H D}\right)$ corresponds to the MHD-scale $\mathbf{E}$, resulting from $\mathbf{B}$ being frozen-in to $\mathbf{u}$. The second term on the r.h.s. $\left(\mathbf{E}_{\text {Hall }}\right)$ is the Hall term, which results from differential ion and electron motion and, for $m_{e} / m_{i} \ll 1$, ensures $\mathbf{B}$ remains frozen-in to the electron fluid velocity $\left(\mathbf{u}_{e}\right)$ even at small scales where the ions decouple from $\mathbf{B}$. The third and fourth terms on the r.h.s. are the electron pressure $\left(\mathbf{E}_{P_{e}}\right)$ and electron inertia $\left(\mathbf{E}_{\text {inertia }}\right)$ terms, respectively, which give rise to nonideal $\mathbf{E}$ that allow electrons to decouple from $\mathbf{B}$. The final term on the r.h.s. $\left(\mathbf{E}_{\delta m_{e}}\right)$ contains higher-order, finite-electron-mass corrections. In many systems, $m_{e} / m_{i} \ll 1$ and these higher-order corrections can be neglected.

Previous observational studies of Ohm's law in space plasmas largely focused on specific structures, such as reconnecting current sheets. Multipoint measurements from the Cluster (André et al., 2004; Khotyaintsev et al., 2006) and, more recently, Magnetospheric Multiscale (MMS) (Genestreti 
et al., 2018; Macek et al., 2019; Shuster et al., 2019; Torbert et al., 2016; Webster et al., 2018) missions have directly observed $\mathbf{E}_{\text {Hall }}$ at small-scale current sheets and revealed the nonideal $\mathbf{E}$, which enables magnetic reconnection, is mainly associated with $\mathbf{E}_{P_{e}}$ with a weaker contribution from $\mathbf{E}_{\text {inertia }}$. These results are in agreement with previous studies of laboratory reconnection (Brown et al., 2006; Cothran et al., 2005).

In the context of turbulence, the spectrum of $\mathbf{E}$ is observed to undergo a change in power law near ion scales, with a steeper power law, close to that of $\mathbf{B}$ or $\mathbf{u}$, at large scales and a shallower power law at sub-ion scales, across a variety of space plasmas (Bale et al., 2005; Breuillard et al., 2018; Chen et al., 2011; Ergun et al., 2015; Sahraoui et al., 2009; Stawarz et al., 2016). The change in power law is linked to the interplay between the terms in Ohm's law at different scales in the plasma (e.g., Matteini et al., 2017; Narita et al., 2019); however, direct analysis of how Ohm's law shapes the $\mathbf{E}$ spectrum has been limited to numerical and theoretical studies. Two-fluid, hybrid, and full particle-in-cell (PIC) simulations of homogeneous turbulence show that, while the large-scale spectrum is dominated by $\mathbf{E}_{M H D}$, the small-scale spectrum is shaped by a combination of $\mathbf{E}_{\text {Hall }}$ and $\mathbf{E}_{P_{e}}$, which both exhibit similar power laws, with possible contributions from $\mathbf{E}_{\text {inertia }}$ in the case of full PIC (Franci et al., 2015; González et al., 2019).

The high-resolution, three-dimensional, multispacecraft measurements from MMS (Burch et al., 2016), which allow the computation of nearly all the terms in generalized Ohm's law down to scales approaching those of the electrons, make it uniquely suited for examining Ohm's law within turbulent plasmas. In this study, we explore the power spectra of the terms in generalized Ohm's law using MMS observations of turbulence in Earth's magnetosheath. In contrast to previous observational studies of Ohm's law, which examined individual small-scale structures, this study provides a statistical picture of Ohm's law across the ensemble of multiscale structures and fluctuations that are excited within the turbulent plasma. Sections 2 and 3 provide an overview of the data set and considerations regarding the analysis. Section 4.1 examines the spectra and relative importance of the terms in generalized Ohm's law. Section 4.2 examines how the terms combine to form the total E. Section 4.3 examines the relative importance of linear and nonlinear dynamics.

\section{Data Set}

We focus on three intervals of high-resolution "burst" magnetosheath data observed by MMS. B measurements are provided by the Fluxgate (FGM) and Searchcoil (SCM) magnetometers (Le Contel et al., 2016; Russell et al., 2016) at 128 and 8192 vectors/s, respectively. Three-dimensional E measurements are provided by the Electric Field Double Probes (EDP) at 8192 vectors/s (Ergun et al., 2016; Lindqvist et al., 2016). Ion and electron particle moments at 0.15 and 0.03 -s resolution, respectively, are provided by the Fast Plasma Investigation (FPI) (Pollock et al., 2016). The single-fluid velocity is computed from the data as $\mathbf{u}=\left(m_{i} \mathbf{u}_{i}+m_{e} \mathbf{u}_{e}\right) /\left(m_{i}+m_{e}\right)$ with $\mathbf{u}_{e}$ averaged to the ion time resolution, since $\mathbf{u}$ is dominated by $\mathbf{u}_{i}$ at the observable scales. The current is computed from the FPI measurements as $\mathbf{j}=e n_{e}\left(\mathbf{u}_{i}-\mathbf{u}_{e}\right)$, where quasineutrality is employed, allowing the use of the higher time resolution $n_{e}$ measurement, and $\mathbf{u}_{i}$ is interpolated to the electron time resolution since the small-scale current is observed to be dominated by $\mathbf{u}_{e}$. The current can additionally be computed from the curl of $\mathbf{B}$ using the multispacecraft curlometer technique (Robert et al., 1998). For both $\mathbf{u}$ and $\mathbf{j}$, the ions are taken to be protons.

The time periods and average plasma properties for the intervals, referred to as I1, I2, and I3, are provided in Table 1 and overviews of $\mathbf{B}, \mathbf{u}$, and $\mathbf{E}$ for the intervals are shown in Figures $1 \mathrm{a}-1 \mathbf{j}$. With $\langle\ldots\rangle$ denoting a temporal average over the interval, the average density $\left(n_{0} \equiv\left\langle n_{e}\right\rangle\right)$, temperature for species $s\left(T_{s 0} \equiv\left\langle T_{s}\right\rangle\right)$, magnetic field strength $\left(B_{0} \equiv|\langle\mathbf{B}\rangle|\right)$, and root-mean-square fluctuation amplitude $\left(\delta b_{r m s} \equiv \sqrt{\left\langle|\mathbf{B}-\langle\mathbf{B}\rangle|^{2}\right\rangle}\right)$ are used to define the inertial lengths $\left(d_{s} \equiv \sqrt{m_{s} / \mu_{0} e^{2} n_{0}}\right)$, gyroradii $\left(\rho_{s} \equiv \sqrt{2 m_{s} k_{B} T_{s 0} / e B_{0}^{2}}\right)$, plasma beta $\left(\beta_{s} \equiv 2 \mu_{0} n_{0} k_{B} T_{s 0} / B_{0}^{2}\right)$, Alfvén speed $\left(V_{A} \equiv B_{0} / \sqrt{\mu_{0} m_{i} n_{0}}\right)$, and turbulence amplitudes $\left(\delta b_{r m s} / B_{0}\right)$, where $\mu_{0}$ is the vacuum permeability and $k_{B}$ is the Boltzmann constant. These intervals are selected because they have some of the smallest MMS separations $(\sim 6 \mathrm{~km})$, providing access to terms requiring multispacecraft gradients well into the sub-ion scales, and covered a range of $\beta_{i}$ and $\beta_{e}$. The elongation and planarity of the MMS formation ranged from $0.09-0.24$ and $0.16-0.35$, respectively, making it well suited for gradient computation 
Table 1

Average Spacecraft Formation, Plasma, and Fluctuation Properties

\begin{tabular}{|c|c|c|c|c|c|c|c|c|c|c|c|}
\hline ID & Time interval (UTC) & MMS separation $(\mathrm{km})$ & $U_{0}(\mathrm{~km} / \mathrm{s})$ & $V_{A}(\mathrm{~km} / \mathrm{s})$ & $\rho_{i}(\mathrm{~km})$ & $d_{i}(\mathrm{~km})$ & $\rho_{e}(\mathrm{~km})$ & $d_{e}(\mathrm{~km})$ & $\beta_{i}$ & $\beta_{e}$ & $\delta b_{\text {rms }} / B_{0}$ \\
\hline I1 & 2016-09-28/16:50:14-17:03:31 & 6.9 & 310 & 170 & 180 & 75 & 0.99 & 1.8 & 5.8 & 0.32 & 1.2 \\
\hline I2 & 2016-12-09/09:01:40-09:07:00 & 6.1 & 230 & 97 & 180 & 49 & 1.3 & 1.2 & 13 & 1.2 & 1.3 \\
\hline I3 & 2017-01-28/09:05:25-09:11:12 & 5.6 & 150 & 50 & 290 & 48 & 2.2 & 1.1 & 37 & 3.9 & 1.9 \\
\hline
\end{tabular}

Abbreviation: MMS, Magnetospheric Multiscale.

(Robert et al., 1998). For all of the intervals, $\delta b_{r m s} / B_{0}>1$. I2 and I3 are located near the subsolar point at (11, $3,0.3) R_{E}$ and $(11,-3,2) R_{E}$, respectively, in Geocentric Solar Ecliptic (GSE) coordinates and I1 is toward the flank at $(2,11,-0.5) R_{E}$.
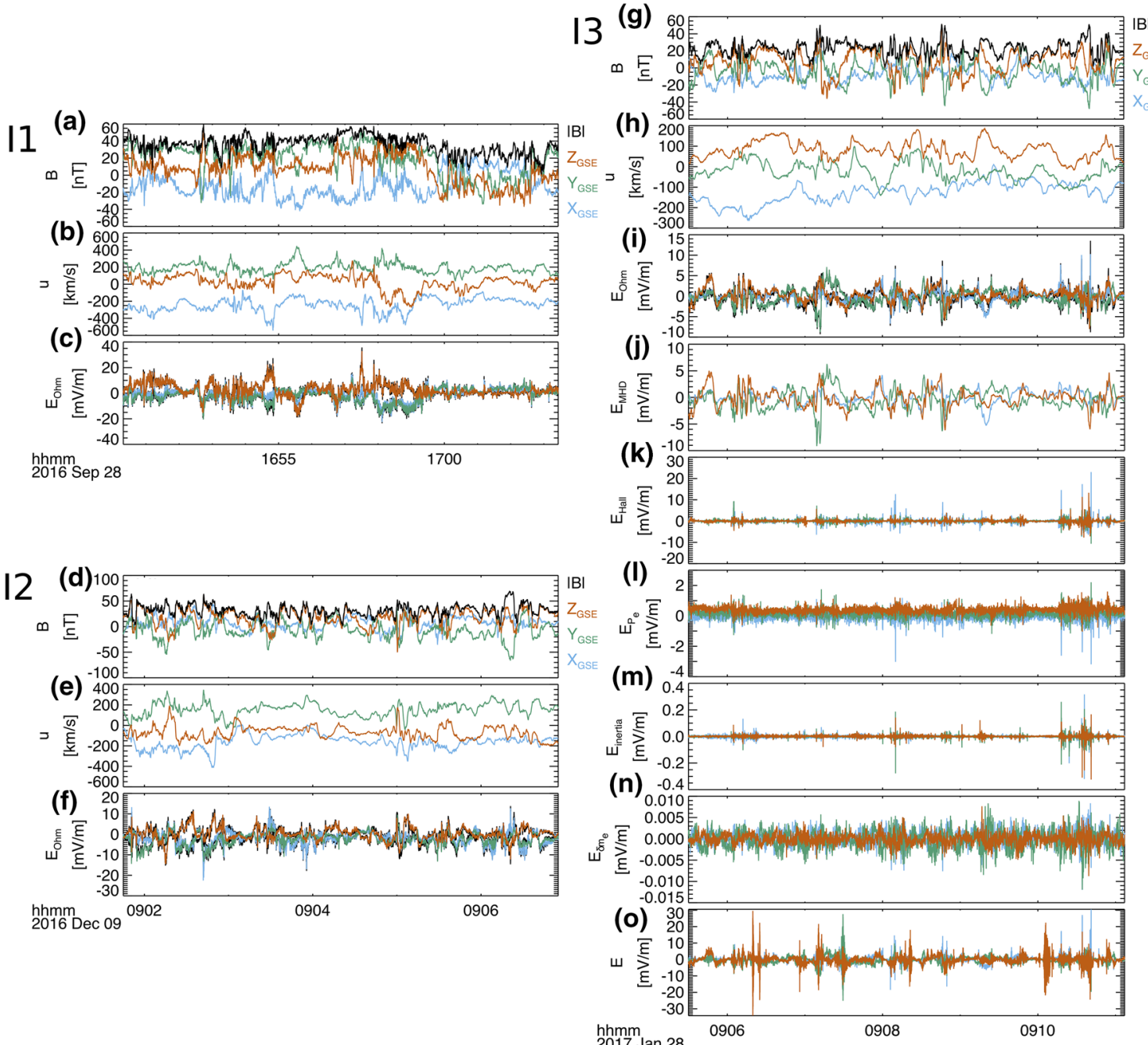

Figure 1. Overview of turbulent magnetosheath intervals examined in this study. (a-i) B, $\mathbf{u}$, and $\mathbf{E}$ in GSE coordinates for intervals I1-I3. B and $\mathbf{u}$ are provided as measured by MMS1, while $\mathbf{E}$ is given as the sum of the computed Ohm's law terms at the barycenter of the MMS formation with the measured $\mathbf{E}_{b a r y}$ from EDP at the same time resolution plotted behind in black. ( $\mathrm{j}-0$ ) Computed Ohm's law terms and highest resolution E from EDP for I3 in GSE coordinates. Single-spacecraft measurements from MMS1 are given in panels $(\mathrm{j}),(\mathrm{k})$, and $(\mathrm{o})$, while $(1-n)$ provide measurements at the barycenter of the MMS formation. EDP, Electric Field Double Probes; GSE, Geocentric Solar Ecliptic; MMS, Magnetospheric Multiscale. 

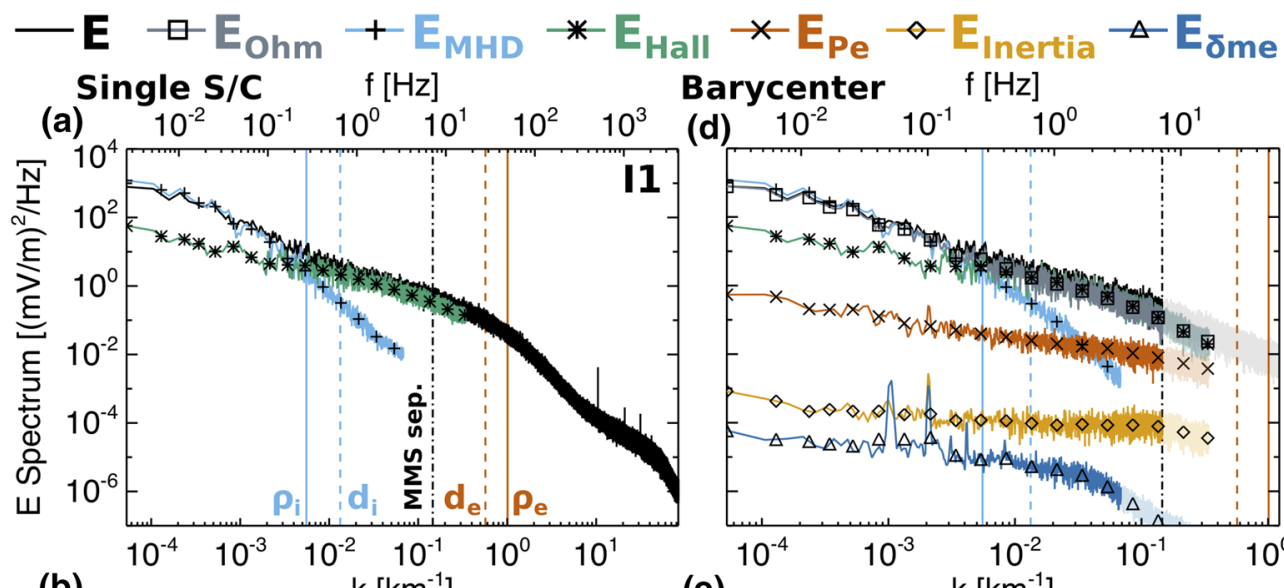

(d) $\begin{array}{llll}10^{-2} & 10^{-1} & 10^{0} & 10^{1}\end{array}$

(b)

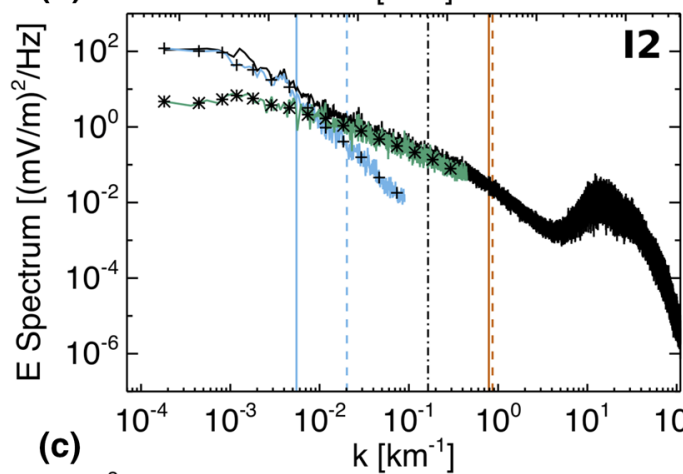

(e)

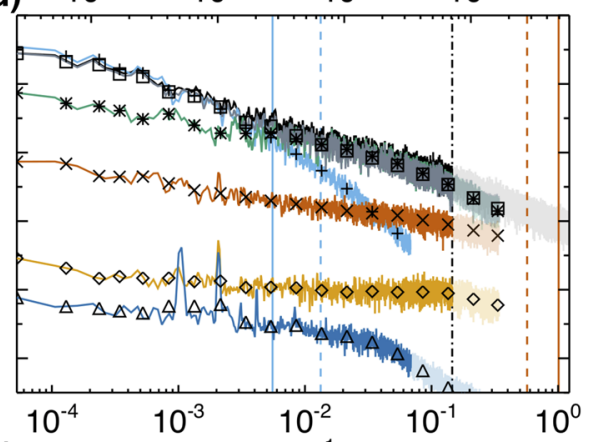

e)

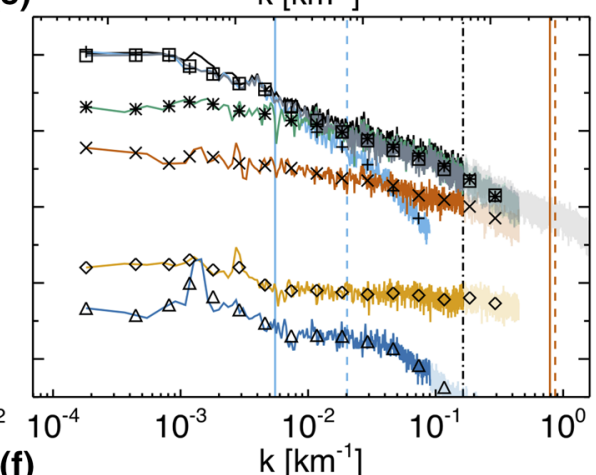

(f)
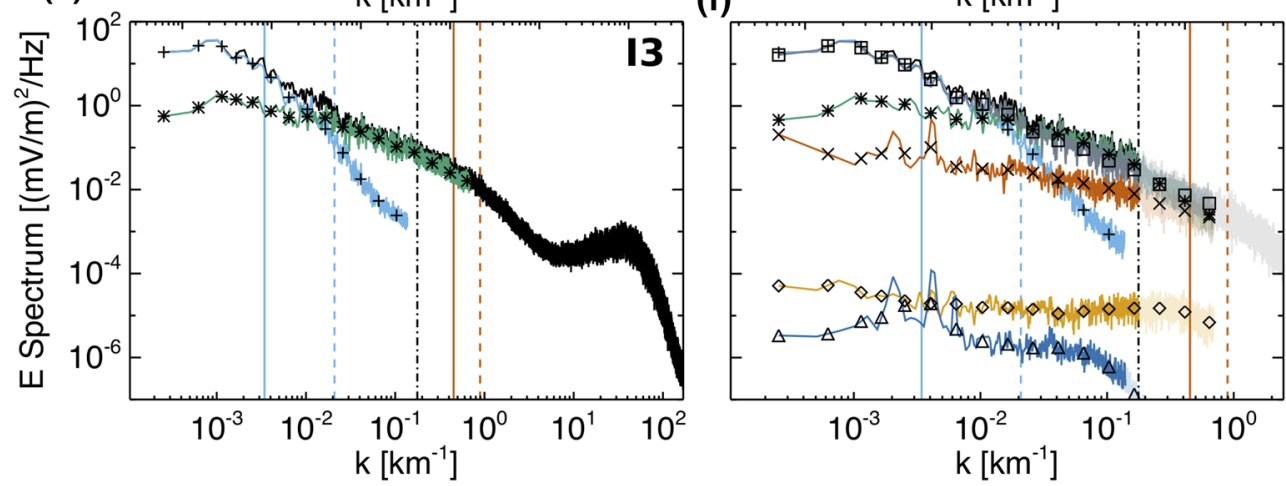

Figure 2. (a-c) Omnidirectional power spectra of the measured $\mathbf{E}_{1 S C}$ and the Ohm's law terms available from single-spacecraft measurements $\left(\mathbf{E}_{M H D, 1 S C}\right.$ and $\left.\mathbf{E}_{H a l l, 1 S C}\right)$ in the background flow frame for I1-I3. Spectra from the four spacecraft are averaged together. (d-f) Omnidirectional power spectra of the barycenter $\mathbf{E}_{b a r y}$ and Ohm's law terms, as well as the power spectrum of the sum of the Ohm's law terms in the background flow frame for I1-I3. Faded portions of the curves indicate the scales expected to be filtered due to multispacecraft averaging or gradient computation. The sudden decrease in $\mathbf{E}_{\delta m_{e}}$ power is associated with $\mathbf{p}_{i}$ measurements reaching the noise level and faded portions of the curve indicate scales smaller than the ion resolution. Wavenumbers along the $\mathbf{U}_{0}$ direction are estimated from the Taylor hypothesis and vertical lines denote wavenumbers associated with ion and electron plasma length scales and the MMS separation. Symbols show spectra averaged in wavenumber over 1/5th of a decade. MMS, Magnetospheric Multiscale.

The validity of the Taylor hypothesis (Taylor, 1938) using the average flow velocity $\left(U_{0} \equiv|\langle\mathbf{u}\rangle|\right)$ is verified at the spacecraft separation by comparing second-order magnetic structure functions computed using the Taylor hypothesis to the six unique spacecraft pairs in the MMS formation (Chasapis et al., 2017; Chen \& Boldyrev, 2017; Chhiber et al., 2018; Stawarz et al., 2019). The ratio of second-order structure functions are within a factor of 1.27 for the intervals. Given the validity of the Taylor hypothesis at the MMS separation, it is reasonable to assume the Taylor hypothesis also holds at scales comparable to or larger than this scale. 

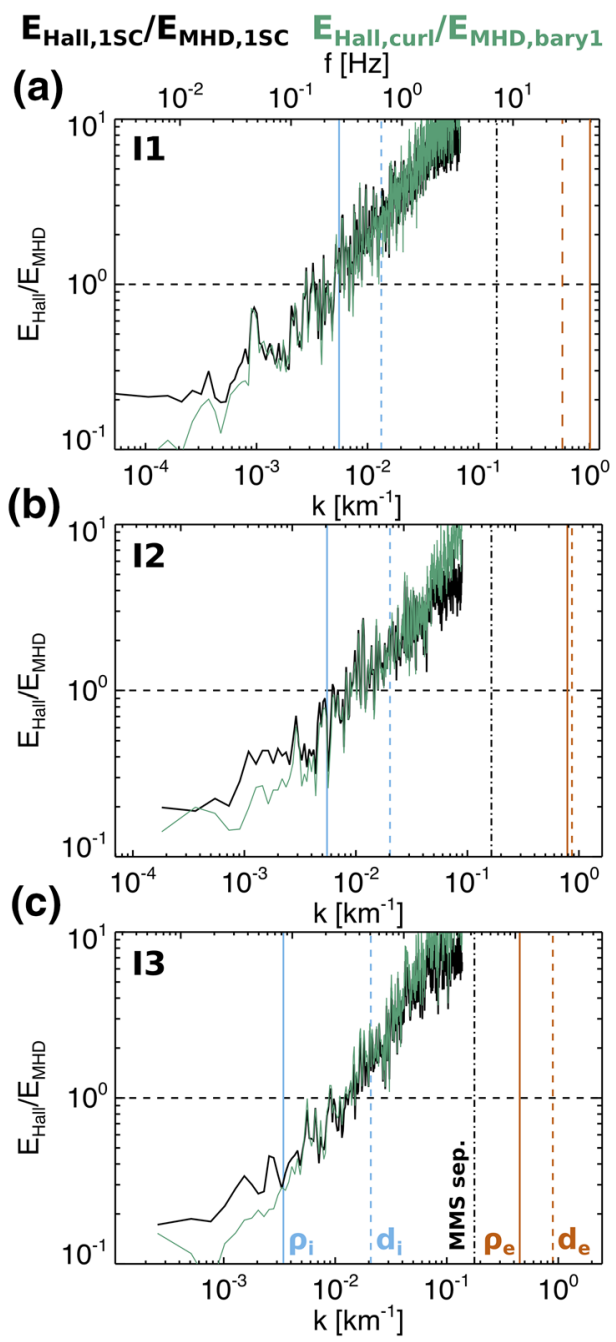

Figure 3. $E_{\text {Hall,1SC }} / E_{M H D, 1 S C}$ (black) and $E_{\text {Hall,curl }} / E_{M H D, \text { bary } 1}$ (green) as a function of scale given by the ratio of Fourier amplitudes in the background flow frame for I1-I3 (a-c, respectively). Wavenumbers along the $\mathbf{U}_{0}$ direction are estimated from the Taylor hypothesis and vertical lines denote wavenumbers associated with ion and electron plasma length scales and the MMS separation. MMS, Magnetospheric Multiscale.

\section{Analysis}

The terms in Equation 1 are computed using a combination of single and multispacecraft techniques. Figures $1 j-10$ give an overview of the Ohm's law terms and measured $\mathbf{E}$ in the time domain for I3. $\mathbf{E}_{M H D}$ and $\mathbf{E}_{\text {Hall }}$ can both be computed from a single spacecraft with the $\mathbf{B}$ measurements averaged to the time resolution of the, respective, $\mathbf{u}$ and $\mathbf{j}$ data. The remaining terms, $\mathbf{E}_{P_{e}}, \mathbf{E}_{\text {inertia }}$, and $\mathbf{E}_{\delta m_{e}}$, require the computation of divergences, which can be done using standard multispacecraft methods (Robert et al., 1998; Shuster et al., 2019). Such methods assume linear gradients over the spacecraft separation, which limits the analysis of these terms to scales larger than the MMS separation. $\mathbf{E}_{\text {iner- }}$ tia additionally involves a time derivative, $\left(m_{e} / e^{2} n\right) \partial_{\mathbf{j}} \mathbf{j}$, which cannot be computed from the data, since, by virtue of the Taylor hypothesis, any apparent temporal fluctuations are purely associated with advected spatial structures. This term is neglected in the computation of $\mathbf{E}_{\text {inertia }}$. In computing $\mathbf{E}_{\delta m_{e}}$, approximately six terms in the infinite sum are needed to converge to double precision accuracy.

A total $\mathbf{E}$ due to the combination of all measurable Ohm's law terms $\left(\mathbf{E}_{\text {Ohm }}\right)$ is computed by averaging $\mathbf{E}_{M H D}$ and $\mathbf{E}_{\text {Hall }}$ to the barycenter of the formation (Figures 1c, 1f and 1i). Several methods can be devised for averaging these terms, including (1) computing $\mathbf{E}_{M H D}$ and $\mathbf{E}_{\text {Hall }}$ for each spacecraft and then averaging, such that $\mathbf{E}_{M H D, \text { bary } 1}=\left\langle\mathbf{E}_{M H D}\right\rangle_{\text {bary }}$ and $\mathbf{E}_{\text {Hall,bary } 1}=\left\langle\mathbf{E}_{\text {Hall }}\right\rangle_{\text {bary }}$, or (2) averaging each variable involved in the computation to the barycenter and then computing the Ohm's law terms, such that $\mathbf{E}_{M H D \text {, bary } 2}=$ $-\langle\mathbf{u}\rangle_{\text {bary }} \times\langle\mathbf{B}\rangle_{\text {bary }}$ and $\mathbf{E}_{\text {Hall, bary } 2}=\langle\mathbf{j}\rangle_{\text {bary }} \times\langle\mathbf{B}\rangle_{\text {bary }} / e\langle n\rangle_{\text {bary }}$, where $\langle\ldots\rangle_{\text {bary }}$ denotes a barycenter average. Additionally, $\mathbf{E}_{\text {Hall }}$ can be computed using the curlometer derived current, such that $\mathbf{E}_{\text {Hall, curl }}=\mathbf{j}_{\text {curl }} \times\langle\mathbf{B}\rangle_{\text {bary }} / e\langle n\rangle_{\text {bary }}$. Method 1 is taken to be the nominal method of performing the barycenter averaging in this study unless otherwise noted, however, each of these methods have been examined and produce consistent results down to the scale of the spacecraft separation. $\mathbf{E}_{M H D}$ is found to be subdominant at the smallest observed scales (Figure 2), so $\mathbf{E}_{M H D}$ is interpolated to the electron time resolution in computing $\mathbf{E}_{\text {Ohm }}$. The Ohm's law terms at the barycenter of the formation are compared with the measured $\mathbf{E}$ averaged to the barycenter such that $\mathbf{E}_{\text {bary }}=\langle\mathbf{E}\rangle_{\text {bary }}$. In the following analysis, for clarity, the above notation is used when referring specifically to quantities computed using the various barycenter averaging procedures, while a subscript $1 S C$ refers to the single-spacecraft measurements. Such notation is omitted when discussing the behavior of the terms in general without regard for

the specific method of computation.

Generally good agreement is found between the timeseries of $\mathbf{E}_{\text {Ohm }}$ and the measured $\mathbf{E}_{\text {bary }}$ when compared at the same time resolution (Figures 1c, 1f, and 1i). However, small differences are observed, particularly in the small-scale structures. Furthermore, as can be seen in the highest resolution $\mathbf{E}$ measurements (Figure 10), additional intense $\mathbf{E}$ activity is present at shorter timescales than those for which the terms in Ohm's law can be computed.

In the remaining analysis, $\mathbf{E}$ is examined in the inertial reference frame moving with the uniform background plasma flow in accordance with the Lorentz transformation, such that $\mathbf{E}_{p l 0}=\mathbf{E}_{s c}+\mathbf{U}_{0} \times \mathbf{B}$, where $\mathbf{E}_{p l 0}$ in the electric field in the frame moving with the average plasma flow and $\mathbf{E}_{s c}$ is the electric field measured by the spacecraft (Chen et al., 2011; Howes et al., 2014). As $\mathbf{E}_{\text {plo }}$ is examined throughout the analysis, we suppress the subscript $p l 0$ in the remaining discussion. Unlike the Galilean frame transformation of $\mathbf{u}$, the frame transformation of $\mathbf{E}$ not only introduces a frame dependent background 

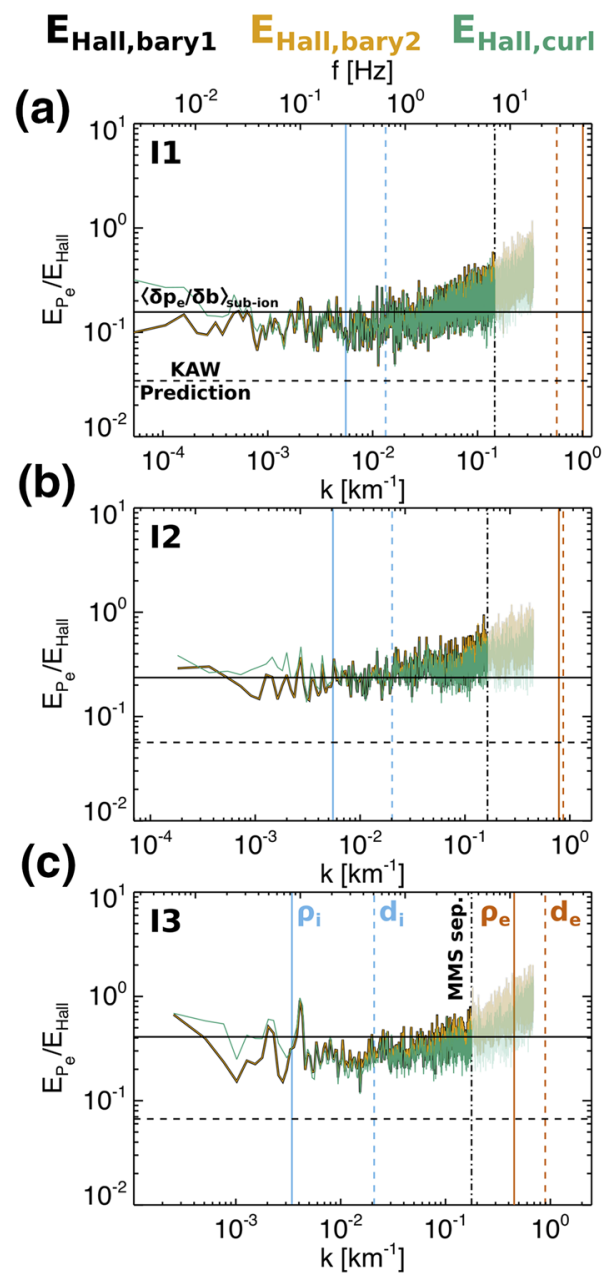

Figure 4. $E_{P_{e}} / E_{\text {Hall }}$ as a function of scale given by the ratio of Fourier amplitudes for I1-I3 (a-c, respectively) computed using $\mathbf{E}_{\text {Hall,bary } 1}$ (black), $\mathbf{E}_{\text {Hall,bary2 }}$ (yellow), and $\mathbf{E}_{\text {Hall,curl }}$ (green). Horizontal lines denote the expected ratio based on the observed average ratio of sub-ion scale $\mathbf{B}$ and $\mathbf{p}_{e}$ fluctuations (solid) and based on linear kinetic Alfvén waves (dashed). Wavenumbers along the $\mathbf{U}_{0}$ direction are estimated from the Taylor hypothesis and vertical lines denote wavenumbers associated with ion and electron plasma length scales and the MMS separation. MMS, Magnetospheric Multiscale.
$\mathbf{E}_{0}=\mathbf{U}_{0} \times \mathbf{B}_{0}$, but also a frame dependent electric fluctuation, $\delta \boldsymbol{E}=\mathbf{U}_{0} \times \delta \mathbf{b}$, associated with the advection of magnetic fluctuations past the spacecraft. The $\mathbf{E}$ fluctuation that remains after the frame transformation corresponds to the electric fields induced by the turbulent plasma motions. Within Ohm's law, $\mathbf{E}_{M H D}$ is frame dependent and introduces the frame dependance into $\mathbf{E}$, while the remaining terms are independent of frame.

\section{Results}

\subsection{Ohm's Law Spectra}

Figures $2 \mathrm{a}-2 \mathrm{f}$ show omnidirectional power spectra (i.e., trace of the spectral tensor) for the five Ohm's law terms, $\mathbf{E}_{\text {Ohm }}$, and the measured $\mathbf{E}$ for all intervals in the frame moving with $\mathbf{U}_{0}$. Since barycenter averaging filters power from the fluctuations at scales smaller than the MMS separation and single-spacecraft electron measurements provide information at scales smaller than the MMS separation, Figures $2 \mathrm{a}-2 \mathrm{c}$ provide the single-spacecraft spectra for $\mathbf{E}_{1 S C}, \mathbf{E}_{M H D, 1 S C}$, and $\mathbf{E}_{H a l l, 1 S C}$, while Figures $2 \mathrm{~d}-2 \mathrm{f}$ provide spectra for all terms at the barycenter. Single-spacecraft spectra are averaged across the four spacecraft after the spectrum is computed, while barycenter averaged spectra are computed by averaging to the barycenter as discussed in Section 3 prior to computing the spectra.

The spectral power laws in all three intervals show features consistent with previous studies of magnetosheath turbulence. At scales larger than the ion scales, the $\mathbf{E}$ and $\mathbf{B}$ (not shown) spectral power laws are similar to each other and shallower than $\sim k^{-5 / 3}$, as is typical in the magnetosheath (Huang et al., 2017). At sub-ion scales, the E spectrum follows $\sim k^{-0.8}$ and a corresponding $\sim k^{-2.8}$ power law is present in the magnetic spectrum, which are typical of turbulent electromagnetic field spectra (e.g., Breuillard et al., 2018; Huang et al., 2017). Additionally, I 2 and I3 both show a further steepening of the magnetic spectrum to $\sim k^{-3.2}$ at scales several times larger than $d_{e}\left(\sim 0.1 d_{i}\right)$, as reported previously for I2 (Stawarz et al., 2019). The $\mathbf{E}$ spectrum undergoes a similar steepening such that it maintains the factor of $\sim k^{2}$ relative to the magnetic spectrum.

From the single-spacecraft spectra, $\mathbf{E}_{M H D, 1 S C}$ provides the dominant contribution to $\mathbf{E}_{1 S C}$ at scales larger than $\sim d_{i}$ and $\mathbf{E}_{H a l l, 1 S C}$ appears to be the dominant contribution from $\sim d_{i}$ to scales between $\rho_{e}$ and $d_{e}$ at the greatest extent (I3; Figure 2c). Figures 2d-2f support the single-spacecraft results up to the MMS separation and additionally reveal that a subdominant, but nonzero, contribution to the sub-ion scale $\mathbf{E}$ comes from $\mathbf{E}_{P_{e}}$. As expected for these scales and from the fact that $m_{e} / m_{i} \ll 1$, the measurable $\mathbf{E}_{\text {inertia }}$ and $\mathbf{E}_{\delta m_{e}}$ terms are much smaller than the other terms and, therefore, make little contribution to $\mathbf{E}_{\mathrm{Ohm}}$.

To understand the interplay between the dominant terms, consider the dimensionless Ohm's law, including $\mathbf{E}_{M H D}, \mathbf{E}_{\text {Hall }}$, and $\mathbf{E}_{P_{e}}$, given by

$$
\frac{\mathbf{E}}{V_{A} B_{0}}=-\frac{\delta \mathbf{u}}{V_{A}} \times \frac{\mathbf{B}}{B_{0}}+\frac{d_{i}}{n / n_{0}}\left[\left(\nabla \times \frac{\delta \mathbf{b}}{B_{0}}\right) \times \frac{\mathbf{B}}{B_{0}}-\frac{\beta_{e}}{2} \nabla \cdot \frac{\delta \mathbf{p}_{e}}{n_{0} k_{B} T_{e 0}}\right],
$$



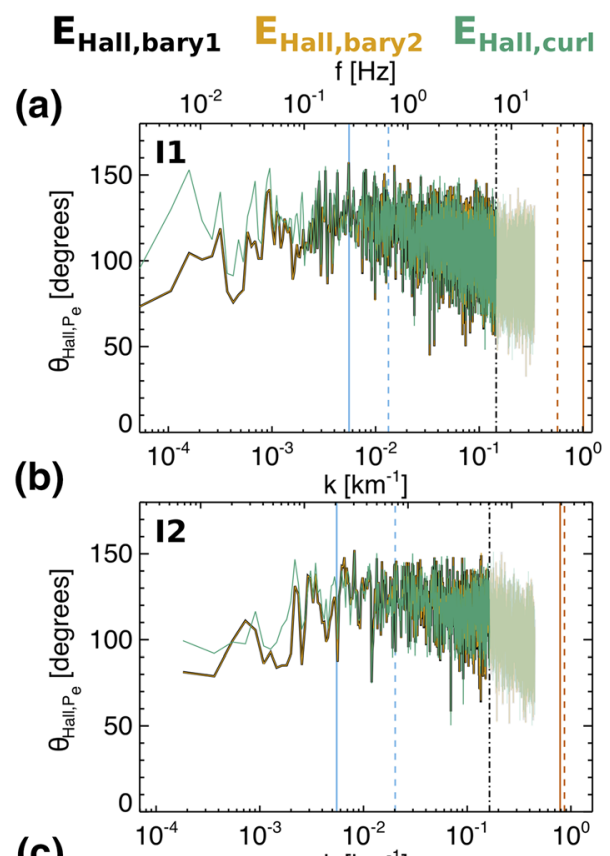

(c)

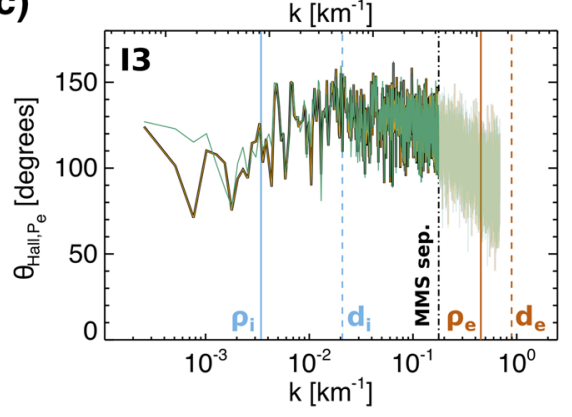

Figure 5. $\theta_{\text {Hall }, P_{e}}$ in Fourier space computed from the cross-spectrum of $\mathbf{E}_{\text {Hall }}$ and $\mathbf{E}_{P_{e}}$ for I1-I3 (a-c, respectively). $\mathbf{E}_{\text {Hall }}$ is averaged to the barycenter of the formation as $\mathbf{E}_{\text {Hall, bary } 1}$ (black), $\mathbf{E}_{\text {Hall,bary } 2}$ (yellow), and $\mathbf{E}_{\text {Hall,curl }}$ (green). Wavenumbers along the $\mathbf{U}_{0}$ direction are estimated from the Taylor hypothesis and vertical lines denote wavenumbers associated with ion and electron plasma length scales and the MMS separation. MMS, Magnetospheric Multiscale.
In Figure 3, the transition between $\mathbf{E}_{M H D}$ and $\mathbf{E}_{\text {Hall }}$ dominated regions of the spectrum occurs in the vicinity of the ion scales for all intervals. For I2 and I3, the transition occurs closer to $d_{i}$, while, in I1, the transition occurs closer to $\rho_{i}$. From Equation 3, the scale of the transition is expected to occur at $k d_{i} \delta v_{A} / \delta u \sim 1$, where $\delta v_{A}=\delta b / \sqrt{\mu_{0} m_{i} n_{0}}$. For Alfvénic fluctuations, it is expected the transition will occur at $k d_{i} \sim 1$; however, in general, the transition depends on the relative fluctuation amplitudes of $\mathbf{B}$ and $\mathbf{u}$. The relative amplitude of root-mean-square magnetic to velocity fluctuations is larger in I1 than in I2 or I3, with values of 1.6, 1.2, and 1.3, respectively, which appears to account for the location of the transition in the intervals.

$\mathbf{E}_{P_{e}}$ scales similarly to $\mathbf{E}_{\text {Hall }}$ with wavenumber up to the MMS separation, particularly for I2 and I3 (Figures $4 \mathrm{~b}$ and $4 \mathrm{c}$ ), consistent with predictions based on the scaling of density and magnetic fluctuations (Franci et al., 2015; Matteini et al., 2017). In I1 (Figure 4a), which has the smallest relative contribution from $\mathbf{E}_{P_{e}}$, there may be a slight difference in scaling with the ratio of amplitudes going as $\sim k^{1 / 3}$ at sub-ion scales. $E_{P_{e}} / E_{\text {Hall }}$ ranges from 0.1 to 0.3 for all three intervals. From Equation 3, $E_{P_{e}} / E_{\text {Hall }} \sim\left(\beta_{e} / 2\right)\left(\delta p_{e} / n_{0} k_{B} T_{e 0}\right) /\left(\delta b / B_{0}\right)$, which is akin to a characteristic $\beta_{e} / 2$ fluctuation, although not identical to that which would be estimated from a locally defined $\beta_{e} / 2$. One might expect $E_{P_{e}} / E_{\text {Hall }}$ to be large for intervals with large $\beta_{e}$, such as I2 and I3. However, this expectation neglects the relative amplitudes of $\delta \mathbf{b} / B_{0}$ and $\delta \mathbf{p}_{e} /\left(n_{0} k_{B} T_{e 0}\right)$, which can introduce a further dependence on $\beta=\beta_{i}+\beta_{e}$.

In the case of linear kinetic Alfvén waves (KAWs) with isothermal $T_{e}$, the relative amplitudes of magnetic and electron pressure fluctuations are given by (Boldyrev et al., 2013)

$$
\frac{\delta p_{e} / n_{0} k_{B} T_{e 0}}{\delta b / B_{0}}=\frac{\delta n / n_{0}}{\delta b / B_{0}}=\left(\frac{\beta^{2}}{2}+\frac{\beta}{2}\right)^{-1 / 2} .
$$

KAW predictions for $E_{P_{e}} / E_{\text {Hall }}$ are indicated as horizontal dashed lines in Figure 4, which tend to underestimate the observed values, indicating an enhanced level of compressive fluctuations relative to the purely linear KAW dynamics. This underestimate may result from modifications due to strong nonlinearities (see Section 4.3), compressive effects, additional wave modes, and/or the presence of coherent structures.

The observed ratio of the $\mathbf{B}$ spectrum to the isotropic electron pressure $\left(p_{e} \equiv \operatorname{Tr}\left[\mathbf{p}_{e}\right] / 3\right.$ ) spectrum (not shown) is roughly constant at sub-ion scales in all intervals. Solid horizontal lines in Figure 4 show the expected $E_{P_{e}} / E_{\text {Hall }}$ for I1-I3 using the observed average ratio $\left(\delta p_{e} / n_{0} k_{B} T_{e 0}\right) /(\delta b /$ $\left.B_{0}\right)$ at sub-ion scales. This prediction does not involve any information about the wavevectors or their alignments, which are included in the full computation of $E_{P_{e}} / E_{\text {Hall }}$. Even so, reasonably good agreement with the observed $E_{P_{e}} / E_{\text {Hall }}$ from the Ohm's law computations is obtained, consistent with the enhanced level of compressibility relative to pure KAW dynamics. Further taking the observed $\left(\delta n / n_{0}\right) /\left(\delta b / B_{0}\right)$ at sub-ion scales as a proxy for $\left(\delta p_{e} / n_{0} k_{B} T_{e 0}\right) /\left(\delta b / B_{0}\right)$, gives similar values for $E_{P_{e}} / E_{\text {Hall }}$, indicating that the isothermal approximation used in the KAW prediction is not responsible for the discrepancy.

\subsection{Total Electric Field}

As seen in Figures 2d-2f, the power in $\mathbf{E}_{\text {Ohm }}$ tends to be slightly smaller than the power in $\mathbf{E}_{\text {Hall, bary } 1}$ at subion scales in all of the intervals. The largest effect is present in I3, which has the largest contribution from 

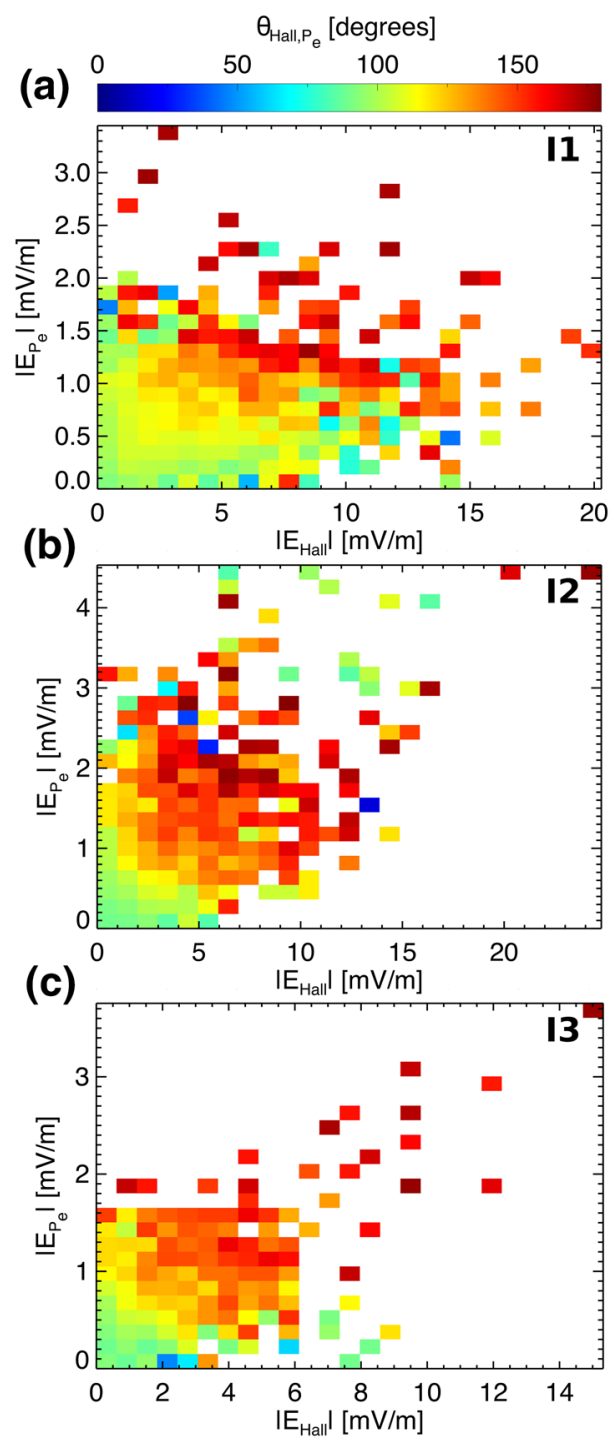

Figure 6. 2D distribution of the average $\theta_{\text {Hall,Pe }}$ in real space as a function of $\left|\mathbf{E}_{\text {Hall }}\right|$ and $\left|\mathbf{E}_{P_{e}}\right|$, where $\mathbf{E}_{\text {Hall,bary } 1}$ is used to average to the barycenter of the formation, for I1-I3 (a-c, respectively).
$\mathbf{E}_{P_{e}}$; however, similar behavior, where $\mathbf{E}_{\text {Ohm }}$ has less power than $\mathbf{E}_{\text {Hall, bary } 1}$, is also present in I1 and I2, although to a lesser extent, and when using other barycenter averaging procedures. Since the most significant contribution to the overall $\mathbf{E}$ in the sub-ion scales comes from $\mathbf{E}_{\text {Hall }}$ and $\mathbf{E}_{P_{e}}$, this observed decrease in $\mathbf{E}_{\text {Ohm }}$ relative to $\mathbf{E}_{\text {Hall }}$ implies an antialignment between these two terms in Ohm's law.

Defining a scale-dependent angle between $\mathbf{E}_{\text {Hall }}$ and $\mathbf{E}_{P_{e}}\left(\theta_{\text {Hall }, P_{e}}\right)$ using the cross-spectrum of the two vectors confirms this apparent antialignment between $\mathbf{E}_{\text {Hall }}$ and $\mathbf{E}_{P_{e}}$ (Figure 5). At scales larger than $\rho_{i}$ and $d_{i}$, where neither $\mathbf{E}_{\text {Hall }}$ or $\mathbf{E}_{P_{e}}$ make a significant contribution to $\mathbf{E}$, the two vectors are uncorrelated with $\theta_{\text {Hall }, P_{e}} \sim 90^{\circ}$. At scales near $\rho_{i}, \theta_{\text {Hall }, P_{e}}$ begins to increase, implying a partial antialignment between the vectors, until it reaches an approximately constant value at $\theta_{\text {Hall }, P_{e}} \sim 120^{\circ}-140^{\circ}$. $\theta_{\text {Hall }, P_{e}}$ decreases back to an uncorrelated value of $90^{\circ}$ at the MMS separation scale, likely due to the unphysical measurements at scales smaller than the formation size. An antialignment between $\mathbf{E}_{\text {Hall }}$ and $\mathbf{E}_{P_{e}}$ at sub-ion scales can also be inferred from previous hybrid simulations (Franci et al., 2015), although to our knowledge the present study is the first time this property has been examined directly. A similar picture is obtained by examining $\theta_{\text {Hall }, P_{e}}$ in real space (Figure 6), with large amplitude $\mathbf{E}_{\text {Hall }}$ and $\mathbf{E}_{P_{e}}$ structures showing significant antialignment on average. In contrast, small amplitude structures have $\theta_{\text {Hall }, P_{e}} \sim 90^{\circ}$ on average, consistent with random noise or the behavior of the large-scale $\mathbf{E}_{\text {Hall }}$ and $\mathbf{E}_{P_{e}}$ in the spectrum.

From a theoretical perspective, such an antialignment may be expected due to the action of currents supported by $\mathbf{p}_{e}$ through the diamagnetic drift, given by $\mathbf{j}_{p_{e}}=\left(\mathbf{B} \times \nabla \cdot \mathbf{p}_{e}\right) / B^{2}$. In a situation where the currents in the system are entirely provided by $\mathbf{j}_{p_{e}}$, a complete cancellation between $\mathbf{E}_{\text {Hall }}$ and the components of $\mathbf{E}_{P_{e}}$ perpendicular to $\mathbf{B}$ occurs, resulting in an antialignment between the two terms. The presence of parallel electric fields associated with $\mathbf{E}_{P_{e}}$ will tend to result in only a partial antialignment. Additionally, the currents within a realistic turbulent system need not be entirely supported by electron diamagnetic drifts and are likely made up of a combination of structures that are entirely, partially, or not at all supported by $\mathbf{j}_{p_{e}}$. Such currents that are not supported by electron diamagnetic drifts could, for example, be supported by ion diamagnetic drifts or inertial effects. Nonetheless, when averaged across all of these structures, the presence of $\mathbf{j}_{p_{e}}$ will tend to push the system toward partial antialignment between $\mathbf{E}_{\text {Hall }}$ and $\mathbf{E}_{P_{e}}$, particularly at sub-ion scales, where ion motions have less influence on $\mathbf{j}$, as observed.

Overall, the Ohm's law terms agree well with the measured $\mathbf{E}$ spectra across the observable scale range. In terms of the Fourier amplitudes, $\mathbf{E}_{O h m}$ and $\mathbf{E}_{\text {bary }}$ agree to within $20 \%$, with the largest discrepancies occurring as a slight offset in the sub-ion scales. Such discrepancies have been reported in previous analyses of Ohm's law at individual reconnection events and could be related to limitations in the observational analysis or physical processes (Torbert et al., 2016). Measurement uncertainties or barycenter averaging may introduce uncertainties into the Ohm's law analysis. While the process of barycenter averaging significantly filters power from the fluctuations at scales comparable to or smaller than the spacecraft separation, it may also have a smaller impact at somewhat larger scales. Consistent results are observed whether $\mathbf{E}_{\text {Hall }}$ is computed using the FPI or curlometer derived $\mathbf{j}$ down to scales comparable to or smaller than the spacecraft separation. The observed antialignment provides some validation that MMS is measuring a real $\nabla \cdot \mathbf{p}_{e}$ signal since a net correlation with $\mathbf{E}_{\text {Hall }}$ is inconsistent with random noise. We have further verified that in intervals with low $\beta_{e}$ and fluctuation amplitudes, where $\mathbf{E}_{P_{e}}$ is not expected to be well measured due to approaching the noise 

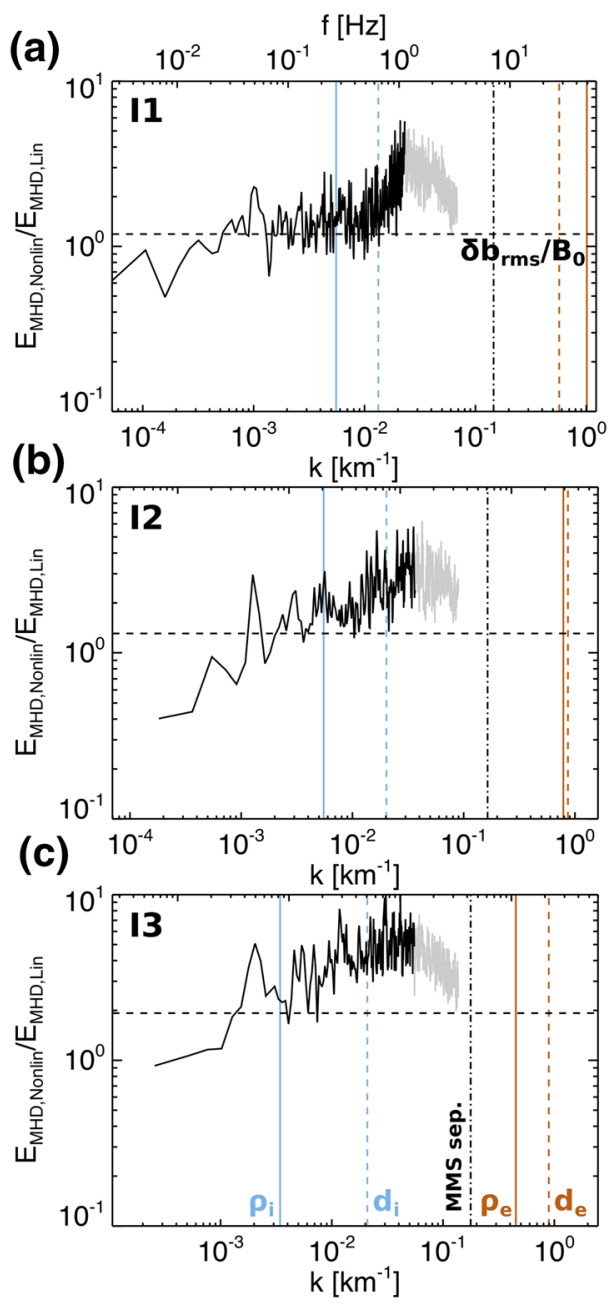

Figure 7. Ratio of the nonlinear and linear contributions to $\mathbf{E}_{M H D, 1 S C}$ as a function of scale in the background plasma frame for I1-I3 (a-c, respectively) with horizontal lines denoting $\delta b_{r m s} / B_{0}$. Faded portions of the curve denote scales contaminated by noise in the ion measurements. Wavenumbers along the $\mathbf{U}_{0}$ direction are estimated from the Taylor hypothesis and vertical lines denote wavenumbers associated with ion and electron plasma length scales and the MMS separation. MMS, Magnetospheric Multiscale. level, many features observed to be consistent with theory and simulations disappear. We, therefore, expect $\mathbf{E}_{\text {Hall }}$ and $\mathbf{E}_{P_{e}}$ to be reasonably computed in the presented intervals; however, such effects may contribute to the observed slight offset.

In terms of physical origins, one possibility may be a finite contribution from the unmeasured time derivative in $\mathbf{E}_{\text {inertia }}$. Recent full PIC turbulence simulations show $\left(m_{e} / e^{2} n\right) \partial_{t} \mathbf{j}$ can make a significantly larger contribution than the other contributions to $\mathbf{E}_{\text {inertia }}$ (González et al., 2019). These simulations use an artificially large $m_{e} / m_{i}$, which enhances the influence of $\mathbf{E}_{\text {inertia }}$, making it difficult to compare these results with the observations directly, and the fact that the Taylor hypothesis appears to work well in these intervals may be an indication that such temporal effects make only a minor impact. However, the fact that the temporal component of $\mathbf{E}_{\text {inertia }}$ can be significantly larger than the measured spatial $\mathbf{E}_{\text {inertia }}$ terms suggests a subdominant influence from $\left(m_{e} / e^{2} n\right) \partial_{\mathbf{j}} \mathbf{j}$ may be possible. Another explanation could be additional contributions to $\mathbf{E}$ that are not captured by the collisionless Ohm's law given in Equation 1, such as enhanced collisionality due to fine-scale structure in the velocity distribution function and anomalous resistivity (Pezzi et al., 2016; Torbert et al., 2016). Either of these processes could have implications for nonideal energy conversion and dissipation in the plasma if they are present.

\subsection{Linear Versus Nonlinear Terms}

Ohm's law contains several of the nonlinearities that give rise to turbulence, particularly those which influence the evolution of the magnetic vector potential and the Lorentz force in the momentum equation. By dividing $\mathbf{B}$ and $\mathbf{u}$ into mean and fluctuating parts, $\mathbf{E}_{M H D}$ and $\mathbf{E}_{\text {Hall }}$ fluctuations can be divided into linear $\left(-\delta \mathbf{u} \times \mathbf{B}_{0}\right.$ and $\delta \mathbf{j} \times \mathbf{B}_{0} /$ en, respectively) and nonlinear $(-\delta \mathbf{u} \times \delta \mathbf{b}$ and $\delta \mathbf{j} \times \delta \mathbf{b} /$ en, respectively) contributions. The mean $\mathbf{B}_{0}$ used in the computation of the linear terms is taken to be the average $\mathbf{B}$ over the whole interval, as opposed to a locally defined average. Since $\mathbf{E}_{M H D}$ and $\mathbf{E}_{\text {Hall }}$ are both accessible using single-spacecraft measurements, the linear and nonlinear contributions are examined using $\mathbf{E}_{M H D, 1 S C}$ and $\mathbf{E}_{\text {Hall,1SC}}$, which are then averaged together after computing the spectra. An additional linear term is also present in $\mathbf{E}_{M H D}$, given by $-\mathbf{U}_{0} \times \delta \mathbf{b}$, which can be removed by a frame transformation into the background flow frame. In the three intervals examined here, the nonlinear terms in both $\mathbf{E}_{M H D}$ and $\mathbf{E}_{\text {Hall }}$ are comparable to or larger than the respective linear terms (Figures 7 and 9), as may be expected from the large values of $\delta b_{r m s} / B_{0}$.

The ratio of nonlinear to linear terms in $\mathbf{E}_{M H D}$ (Figure 7) is roughly $\delta b_{r m s} / B_{0}$ at large scales and then increases to even larger values near $d_{i}$ for I1 and I2 and near $\rho_{i}$ for interval I3. The observed decrease in the ratio for $\mathbf{E}_{M H D}$ at the smallest scales is associated with the ion velocity measurements reaching the noise floor, which can be seen as a flattening of the $\mathbf{E}_{M H D, 1 S C}$ spectra in Figures $2 \mathrm{a}-2 \mathrm{c}$. Since these terms are associated with cross products, the enhancement in the nonlinear term relative to the linear term in $\mathbf{E}_{M H D}$ may indicate a reduction in the average alignment between $\delta \mathbf{u}$ and $\delta \mathbf{b}$ relative to $\delta \mathbf{u}$ and $\mathbf{B}_{0}$. Figure 8 shows that this change in behavior of the nonlinear to linear ratio in $\mathbf{E}_{M H D}$ coincides with a shift toward $90^{\circ}$ of the spectral alignment $\left(\theta_{\delta u, \delta b}\right)$ between $\delta \mathbf{u}$ and $\delta \mathbf{b}$ as computed from the cross spectrum, while the alignment between $\delta \mathbf{u}$ and $\mathbf{B}_{0}$, given by $\theta_{\delta u, B_{0}} \equiv \cos ^{-1}\left(\sqrt{\delta u_{\|}^{2}(k) / \delta u^{2}(k)}\right)$ remains constant as a function of scale. As such, the change in the relative behavior of nonlinear and linear terms in $\mathbf{E}_{M H D}$ is linked to the local alignment properties of the small-scale fluctuations, as opposed to a change in the average alignment of the fluctuations with the background field. This interpretation is compatible with recent MMS observations 

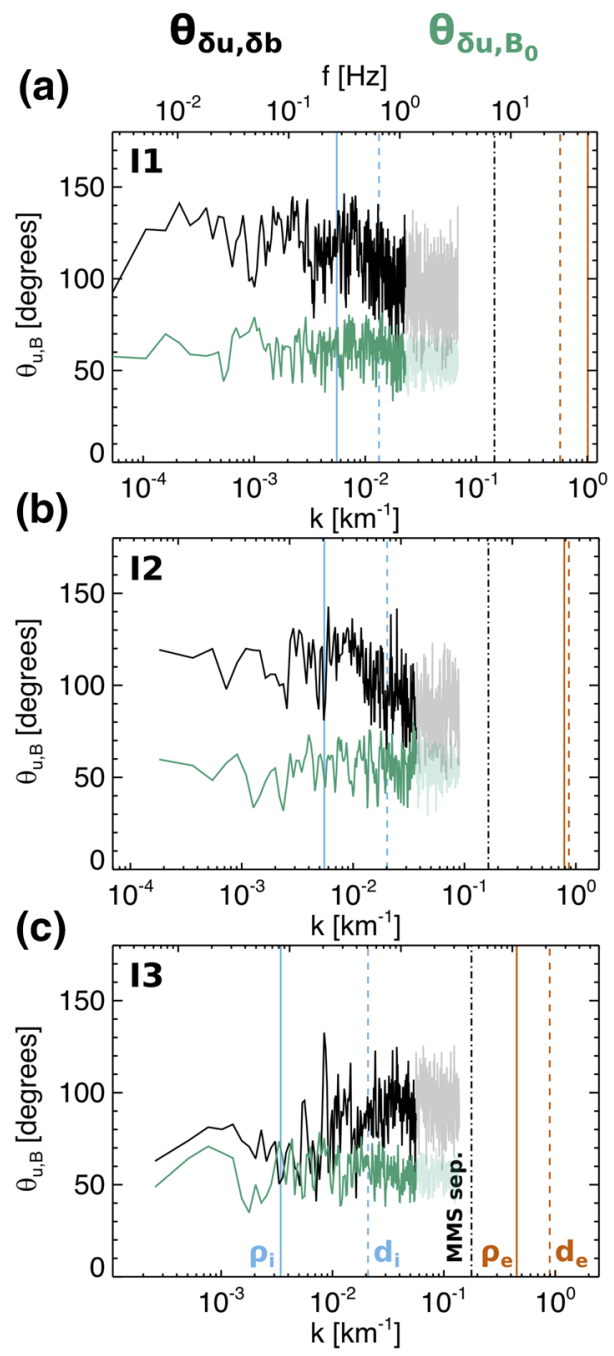

Figure 8. $\theta_{\delta u, \delta b}$ in Fourier space computed from the cross-spectrum of $\delta \mathbf{u}$ and $\delta \mathbf{b}$ (black) and $\theta_{\delta u, B_{0}} \equiv \cos ^{-1}\left(\sqrt{\delta u_{\|}^{2}(k) / \delta u^{2}(k)}\right)$ in Fourier space (green) for I1-I3 (a-c, respectively). $\theta_{\delta u, \delta b}$ can vary from $0^{\circ}$ to $180^{\circ}$, while $\theta_{\delta u, B 0}$ can only vary from $0^{\circ}$ to $90^{\circ}$. Faded portions of the curves denote the scales contaminated by noise in the ion measurements. Wavenumbers along the $\mathbf{U}_{0}$ direction are estimated from the Taylor hypothesis and vertical lines denote wavenumbers associated with ion and electron plasma length scales and the MMS separation. MMS, Magnetospheric Multiscale. of the scale-dependent cross helicity in both the magnetosheath and solar wind (Parashar et al., 2018) and may be linked to the development of magnetic fluctuations parallel to the local field direction by the Hall term as the turbulence transitions into the sub-ion scales (Kiyani et al., 2013). While the observed increase in the importance of the nonlinear $\mathbf{E}_{M H D}$ provides insight into the changing alignment properties of the turbulent fluctuations, it occurs at scales where $\mathbf{E}_{M H D}$ makes a subdominant contribution to the total $\mathbf{E}$. When $\mathbf{E}_{M H D}$ is combined with $\mathbf{E}_{\text {Hall }}$, the relative strength of the nonlinear and linear contributions associated with the $-\mathbf{u}_{e} \times \mathbf{B}$ electric field remains roughly constant with scale.

The ratio of nonlinear to linear terms in $\mathbf{E}_{\text {Hall }}$ (Figure 9) is roughly constant across all observed scales and given by the ratio $\delta b_{r m s} / B_{0} . \mathbf{E}_{\text {Hall }}$ is directly analogous to the Lorentz force in the time evolution of $\mathbf{u}$. The ratio of nonlinear to linear terms in $\mathbf{E}_{\text {Hall }}$, therefore, is related to the linear and nonlinear timescales associated with the turbulent velocity dynamics, although in an incomplete manner as it does not include the advection term. These timescales play a key role in the theoretical description of the turbulent cascade (e.g., Galtier et al., 2000; Goldreich \& Sridhar, 1995; Iroshnikov, 1964; Kolmogorov, 1941; Kraichnan, 1965). The constant ratio between the nonlinear and linear terms for all the intervals in Figure 9 may indicate a balance between the timescales as a function of scale; however, the fact that the ratio appears to exceed one when $\delta b_{r m s} / B_{0}>1$ may make it distinct from the critical balance hypothesis in which a ratio of one would be expected (Chen, 2016; Goldreich \& Sridhar, 1995). A more detailed analysis of the full range of terms appearing in the evolution equations for $\mathbf{u}$ and $\mathbf{B}$ is needed to fully explore this point, which goes beyond the scope of this study.

Interestingly, the ratio of nonlinear to linear terms is scale independent for $\mathbf{E}_{\text {Hall }}$ and is set by $\delta b_{r m s} / B_{0}$, which is a large-scale quantity since $\delta b_{r m s}$ is weighted toward the large-scales for typical turbulent magnetic spectra that are steeper than $k^{-1}$. While it is not unreasonable to expect the ratio of these terms to be linked to $\delta b / B_{0}$ in some manner, the fact that it is set scale-by-scale according to the root-mean-square value is not completely obvious and further theoretical analysis is required to determine the origins and implications of this behavior.

\section{Conclusions}

The role of generalized Ohm's law in shaping the turbulent electric field spectrum from MHD to electron length scales is examined for the first time observationally using the unique capabilities of MMS in Earth's magnetosheath. The results both observationally confirm a number of expectations about the behavior of the terms in generalized Ohm's law, as well as reveal several new features that are relevant for the future theoretical analysis of the small-scale nonlinear dynamics and energy dissipation within collisionless space plasmas.

General expectations about the underlying dynamics at different scales in the plasma are confirmed-namely, $\mathbf{E}_{M H D}$ dominates the dynamics at scales larger than the ion length scales, while $\mathbf{E}_{H a l l}$ and $\mathbf{E}_{P_{e}}$ make more significant contributions at sub-ion scales. The spatial component of $\mathbf{E}_{\text {inertia }}$ remains small over the accessible scales, reaching scales as small as 2.5 to 4 times the larger of $d_{e}$ or $\rho_{e}$. It remains possible that $\mathbf{E}_{\text {inertia }}$ will make a more significant contribution to $\mathbf{E}$ at subelectron scales, as expected from dimensional analysis. The finite-electron-mass corrections to Ohm's law that are expected to be negligible since $m_{e} / m_{i} \ll 1$ are also confirmed to remain small. 

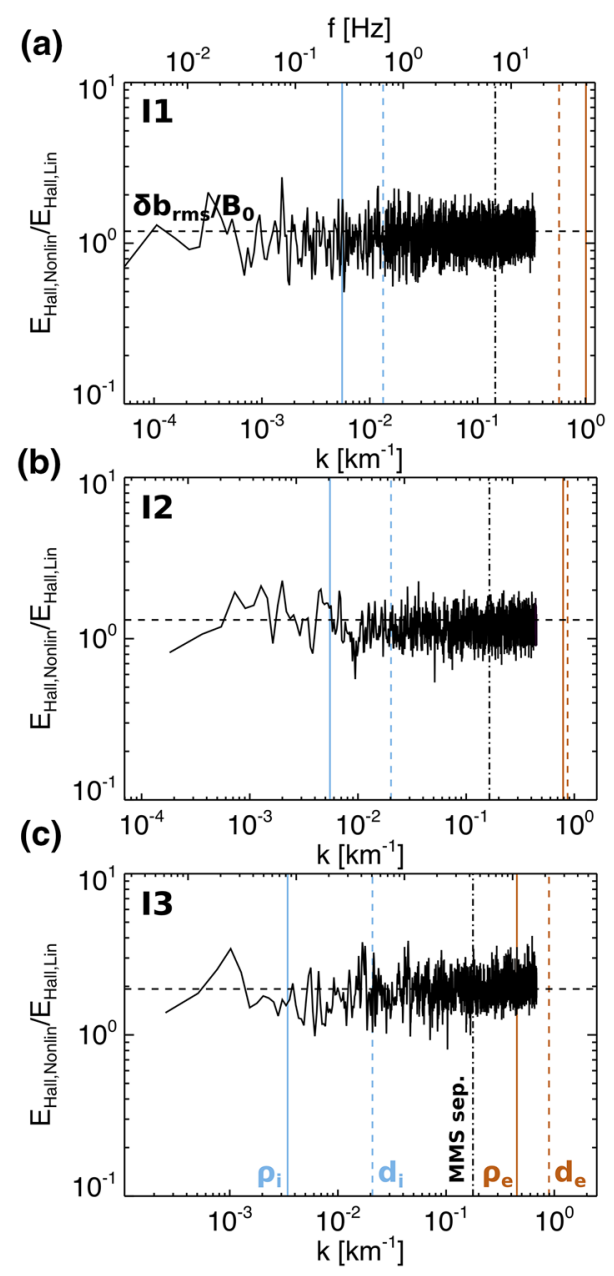

Figure 9. Ratio of the nonlinear and linear contributions to $\mathbf{E}_{\text {Hall,1SC }}$ as a function of scale for I1-I3 (a-c, respectively) with horizontal lines estimate is valid below the spacecraft separation scale. Wavenumbers along the $\mathbf{U}_{0}$ direction are estimated from the Taylor hypothesis and vertical lines denote wavenumbers associated with ion and electron plasma length scales and the MMS separation. MMS, Magnetospheric Multiscale. denoting $\delta b_{r m s} / B_{0}$. Since single-spacecraft measurements are used, the

The interplay of $\mathbf{E}_{\text {Hall }}$ and $\mathbf{E}_{P_{e}}$ at sub-ion scales is examined in detail, revealing these two terms tend to partially antialign. To our knowledge, this is the first direct examination of this aspect of the electric field fluctuations in a turbulent plasma and may relate to the relative role of electron diamagnetic currents within the turbulence, placing constraints on the types of structures or waves that are formed within the plasma. The relative amplitude of $\mathbf{E}_{P_{e}}$ fluctuations are also found to be stronger than expected from purely linear KAWs. $\mathbf{E}_{P_{e}}$ leads to fundamentally different dynamics from $\mathbf{E}_{\text {Hall }}$, in that it generates nonideal electric fields, which allow electrons to decouple from the magnetic field, and is capable of producing a nonzero $\mathbf{j} \cdot \mathbf{E}$. This enhanced $\mathbf{E}_{P_{e}}$ is, therefore, significant when considering the energy transfer and dissipation within turbulent plasmas. Further studies exploring the role of density, anisotropic temperature, and off-diagonal pressure fluctuations in shaping the $\mathbf{E}_{P_{e}}$ spectrum could shed further light on this issue. Previous MMS studies have demonstrated the electron decoupling associated with $\mathbf{E}_{P_{e}}$ is a key factor enabling magnetic reconnection (Genestreti et al., 2018; Torbert et al., 2016). A number of studies have reported reconnecting current sheets within the Earth's magnetosheath, which are thought to be driven by the turbulent fluctuations (e.g., Phan et al., 2018; Retinò et al., 2007; Stawarz et al., 2019; Sundkvist et al., 2007; Vörös et al., 2017; Yordanova et al., 2016). An $\mathbf{E}_{P_{e}}$, which is larger than expected from linear theory, could be a signature of such thin reconnecting current sheets. Further study is needed to determine how such coherent structures factor into the spectral behavior of the turbulent electric field and Ohm's law.

The relative importance of linear and nonlinear contributions to $\mathbf{E}$ are directly examined, demonstrating that in strongly turbulent plasmas, where $\delta b_{r m s} / B_{0}>1$, the nonlinear $\mathbf{E}$ can be the dominant component at both MHD and sub-ion scales. The dominance of the nonlinear $\mathbf{E}$ highlights the need to consider the nonlinear contributions to $\mathbf{E}$ in theoretical descriptions of turbulence under these conditions, which are often found in the magnetosheath, as seen here, and other plasmas, such as the Earth's plasma sheet (Ergun et al., 2018). The analysis of linear and nonlinear terms in $\mathbf{E}$ also reveals information about the changing alignment properties of the magnetic fluctuations as the turbulence transitions into the kinetic scales. While this study focuses on the electric field, a similar analysis of the linear and nonlinear terms governing other variables, such as $\mathbf{B}$ and $\mathbf{u}$, could also be performed using MMS data from the magnetosheath, as has been examined in numerical simulations (Ghosh \& Parashar, 2015a, 2015b). Such an analysis could be used to directly test the critical balance hypothesis (e.g., Chen, 2016; Cho \& Vishniac, 2000; Goldreich \& Sridhar, 1995) in a manner that may be less reliant on definitions of the local magnetic field direction.

J. E. Stawarz and J. P. Eastwood are supported by UKRI/STFC grant ST/ S000364/1 and J. E. Stawarz is additionally supported by the Royal Society University Research Fellowship URF \ R1 $\backslash 201286$. T. N. Parashar was partially supported by Heliophysics Guest Investigator grant 80NSSC19K0284 during this study. L. Franci is supported by UKRI/STFC grant ST/T00018X/1. I. L. Gingell is supported by the Royal Society University Research Fellowship URF $\backslash R 1 \backslash 191547$. The French LPP involvement for the SCM instrument is supported by CNES and CNRS. The authors thank the entire MMS team for their work on the mission.

\section{Data Availability Statement}

Data are publicly available through the MMS Science Data Center (https://lasp.colorado.edu/mms/sdc/ public/) and were analyzed using the SPEDAS software package for IDL (http://spedas.org/blog/).

\section{References}

André, M., Vaivads, A., Buchert, S. C., Fazakerley, A. N., \& Lahiff, A. (2004). Thin electron-scale layers at the magnetopause. Geophysical Research Letters, 31, L03803. https://doi.org/10.1029/2003GL018137

Bale, S. D., Kellogg, P. J., Mozer, F. S., Horbury, T. S., \& Reme, H. (2005). Measurement of the electric fluctuation spectrum of magnetohydrodynamic turbulence. Physical Review Letters, 94, 215002. https://doi.org/10.1103/PhysRevLett.94.215002 
Baumjohann, W., \& Treumann, R. A. (1996). Basic space plasma physics, London, UK: Imperial College Press. https://doi.org/10.1142/p015 Boldyrev, S., Horaites, K., Xia, Q., \& Perez, J. C. (2013). Toward a theory of astrophysical plasma turbulence at subproton scales. The Astrophysical Journal, 777, 41. https://doi.org/10.1088/0004-637X/777/1/41

Borovsky, J. E., Elphic, R. C., Funsten, H. O., \& Thomsen, M. F. (1997). The Earth's plasma sheet as a laboratory for flow turbulence in high-[beta] MHD. Journal of Plasma Physics, 57, 1-34. https://doi.org/10.1017/S0022377896005259

Breuillard, H., Matteini, L., Argall, M. R., Sahraoui, F., Andriopoulou, M., Le Contel, O., \& Cohen, I. J. (2018). New insights into the nature of turbulence in the Earth's magnetosheath using Magnetospheric Multiscale mission data. The Astrophysical Journal, 859, 127. https:// doi.org/10.3847/1538-4357/aabae8

Brown, M. R., Cothran, C. D., \& Fung, J. (2006). Two fluid effects on three-dimensional reconnection in the Swarthmore Spheromak Experiment with comparisons to space dataa). Physics of Plasmas, 13, 056503. https://doi.org/10.1063/1.2180729

Bruno, R., \& Carbone, V. (2013). The solar wind as a turbulence laboratory. Living Reviews in Solar Physics, 10, 2. https://doi.org/10.12942/ lrsp-2013-2

Burch, J. L., Moore, T. E., Torbert, R. B., \& Giles, B. L. (2016). Magnetospheric Multiscale overview and science objectives. Space Science Reviews, 199, 5-21. https://doi.org/10.1007/s11214-015-0164-9

Chasapis, A., Matthaeus, W. H., Parashar, T. N., Fuselier, S. A., Maruca, B. A., Phan, T. D., \& Strangeway, R. J. (2017). High-resolution statistics of solar wind turbulence at kinetic scales using the Magnetospheric Multiscale mission. The Astrophysical Journal Letters, 844 , L9. https://doi.org/10.3847/2041-8213/aa7ddd

Chasapis, A., Matthaeus, W. H., Parashar, T. N., Wan, M., Haggerty, C. C., Pollock, C. J., \& Burch, J. L. (2018). In situ observation of intermittent dissipation at kinetic scales in the earth's magnetosheath. The Astrophysical Journal Letters, 856, L19. https://doi. org/10.3847/2041-8213/aaadf8

Chasapis, A., Yang, Y., Matthaeus, W. H., Parashar, T. N., Haggerty, C. C., Burch, J. L., \& Russell, C. T. (2018). Energy conversion and collisionless plasma dissipation channels in the turbulent magnetosheath observed by the Magnetospheric Multiscale mission. The Astrophysical Journal, 862, 32. https://doi.org/10.3847/1538-4357/aac775

Chen, C. H. K. (2016). Recent progress in astrophysical plasma turbulence from solar wind observations. Journal of Plasma Physics, 82, 535820602. https://doi.org/10.1017/S0022377816001124

Chen, C. H. K., Bale, S. D., Salem, C., \& Mozer, F. S. (2011). Frame dependence of the electric field spectrum of solar wind turbulence. The Astrophysical Journal Letters, 737, L41. https://doi.org/10.1088/2041-8205/737/2/L41

Chen, C. H. K., \& Boldyrev, S. (2017). Nature of kinetic scale turbulence in the Earth's magnetosheath. The Astrophysical Journal, $842,122$. https://doi.org/10.3847/1538-4357/aa74e0

Chen, C. H. K., Klein, K. G., \& Howes, G. G. (2019). Evidence for electron Landau damping in space plasma turbulence. Nature Communications, 10, 740. https://doi.org/10.1038/s41467-019-08435-3

Chhiber, R., Chasapis, A., Bandyopadhyay, R., Parashar, T. N., Matthaeus, W. H., Maruca, B. A., \& Gershman, D. J. (2018). Higher-order turbulence statistics in the Earth's magnetosheath and the solar wind using Magnetospheric Multiscale observations. Journal of Geophysical Research, 123, 9941-9954. https://doi.org/10.1029/2018JA025768

Cho, J., \& Vishniac, E. T. (2000). The anisotropy of magnetohydrodynamic Alfvénic turbulence. The Astrophysical Journal, 539, 273-282. https://doi.org/10.1086/309213

Cothran, C. D., Landreman, M., Brown, M. R., \& Matthaeus, W. H. (2005). Generalized Ohm's law in a 3-D reconnection experiment. Geo physical Research Letters, 32, L03105. https://doi.org/10.1029/2004GL021245

Cranmer, S. R., Asgari-Targhi, M., Miralles, M. P., Raymond, J. C., Strachan, L., Tian, H., \& Woolsey, L. N. (2015). The role of turbulence in coronal heating and solar wind expansion. Philosophical Transactions of the Royal Society A, 373, 20140148. https://doi.org/10.1098/ rsta.2014.0148

Ergun, R. E., Goodrich, K. A., Stawarz, J. E., Andersson, L., \& Angelopoulos, V. (2015). Large-amplitude electric fields associated with bursty bulk flow braking in the Earth's plasma sheet. Journal of Geophysical Research: Space Physics, 120, 1832-1844. https://doi. org/10.1002/2014JA020165

Ergun, R. E., Goodrich, K. A., Wilder, F. D., Ahmadi, N., Holmes, J. C., Eriksson, S., \& Vaivads, A. (2018). Magnetic reconnection, turbulence, and particle acceleration: Observations in the Earth's mgnetotail. Geophysical Research Letters, 45, 3338-3347. https://doi. org/10.1002/2018GL076993

Ergun, R. E., Tucker, S., Westfall, J., Goodrich, K. A., Malaspina, D. M., Summers, D., \& Cully, C. M. (2016). The axial double probe and fields signal processing for the MMS mission. Space Science Reviews, 199, 167-188. https://doi.org/10.1007/s11214-014-0115-X

Falceta-Gonçalves, D., Kowal, G., Falgarone, E., \& Chian, A. C. L. (2014). Turbulence in the interstellar medium. Nonlinear Processes in Geophysics, 21, 587-604. https://doi.org/10.5194/npg-21-587-2014

Franci, L., Landi, S., Matteini, L., Verdini, A., \& Hellinger, P. (2015). High-resolution hybrid simulations of kinetic plasma turbulence at proton scales. The Astrophysical Journal, 812, 21. https://doi.org/10.1088/0004-637X/812/1/21

Galtier, S., Nazarenko, S. V., Newell, A. C., \& Pouquet, A. (2000). A weak turbulence theory for incompressible magnetohydrodynamics. Journal of Plasma Physics, 63, 447-488. https://doi.org/10.1017/S0022377899008284

Genestreti, K. J., Varsani, A., Burch, J. L., Cassak, P. A., Torbert, R. B., Nakamura, R., \& Baumjohann, W. (2018). MMS observation of asymmetric reconnection supported by 3-D electron pressure divergence. Journal of Geophysical Research: Space Physics, 123, 1806-1821. https://doi.org/10.1002/2017JA025019

Gershman, D. J., Viñas, A. F., Dorelli, J. C., Goldstein, M. L., Shuster, J., Avanov, L. A., \& Burch, J. L. (2018). Energy partitioning constraints at kinetic scales in low- $\beta$ turbulence. Physics of Plasmas, 25, 022303. https://doi.org/10.1063/1.5009158

Ghosh, S., \& Parashar, T. N. (2015a). Linear vs. nonlinear acceleration in plasma turbulence. I. Global versus local measures. Physics of Plasmas, 22, 042302. https://doi.org/10.1063/1.4916975

Ghosh, S., \& Parashar, T. N. (2015b). Linear vs. nonlinear acceleration in plasma turbulence. II. Hall-finite-Larmor-radius magnetohydrodynamics. Physics of Plasmas, 22, 042303. https://doi.org/10.1063/1.4916976

Goldreich, P., \& Sridhar, S. (1995). Toward a theory of interstellar turbulence. II. Strong Alfvenic turbulence. The Astrophysical Journal, 438, 763. https://doi.org/10.1086/175121

González, C. A., Parashar, T. N., Gomez, D., Matthaeus, W. H., \& Dmitruk, P. (2019). Turbulent electromagnetic fields at sub-proton scales: Two-fluid and full-kinetic plasma simulations. Physics of Plasmas, 26, 012306. https://doi.org/10.1063/1.5054110

Howes, G. G., Klein, K. G., \& TenBarge, J. M. (2014). Validity of the Taylor hypothesis for linear kinetic waves in the weakly collisional solar wind. The Astrophysical Journal, 789, 106. https://doi.org/10.1088/0004-637X/789/2/106

Huang, S. Y., Hadid, L. Z., Sahraoui, F., Yuan, Z. G., \& Deng, X. H. (2017). On the existence of the Kolmogorov inertial range in the terrestrial magnetosheath turbulence. The Astrophysical Journal Letters, 836, L10. https://doi.org/10.3847/2041-8213/836/1/L10 
Iroshnikov, P. S. (1964). Turbulence of a conducting fluid in a strong magnetic field. Soviet Astronomy, 7, 566.

Kawazura, Y., Barnes, M., \& Schekochihin, A. A. (2019). Thermal disequilibration of ions and electrons by collisionless plasma turbulence. Proceedings of the National Academy of Sciences of the United States of America, 116, 771-776. https://doi.org/10.1073/pnas.1812491116

Khotyaintsev, Y. V., Vaivads, A., Retinò, A., André, M., Owen, C. J., \& Nilsson, H. (2006). Formation of inner structure of a reconnection separatrix region. Physical Review Letters, 97, 205003. https://doi.org/10.1103/PhysRevLett.97.205003

Kiyani, K. H., Chapman, S. C., Sahraoui, F., Hnat, B., Fauvarque, O., \& Khotyaintsev, Y. V. (2013). Enhanced magnetic compressibility and isotropic scale invariance at sub-ion Larmor scales in solar wind turbulence. The Astrophysical Journal, 763, 10. https://doi. org/10.1088/0004-637X/763/1/10

Kolmogorov, A. (1941). The local structure of turbulence in incompressible viscous fluid for very large Reynolds' numbers. Doklady Akademii Nauk SSSR, 30, 301-305 (reprinted in Proc. R. Soc. A, 434, 9-13, 1990).

Kraichnan, R. H. (1965). Inertial-range spectrum of hydromagnetic turbulence. Physics of Fluids, 8, 1385-1387. https://doi. $\operatorname{org} / 10.1063 / 1.1761412$

Le Contel, O., Leroy, P., Roux, A., Coillot, C., Alison, D., Bouabdellah, A., \& de la Porte, B. (2016). The search-coil magnetometer for MMS. Space Science Reviews, 199, 257-282. https://doi.org/10.1007/s11214-014-0096-9

Lindqvist, P. A., Olsson, G., Torbert, R. B., King, B., Granoff, M., Rau, D., \& Tucker, S. (2016). The spin-plane double probe electric field instrument for MMS. Space Science Reviews, 199, 137-165. https://doi.org/10.1007/s11214-014-0116-9

Macek, W. M., Silveira, M. V. D., Sibeck, D. G., Giles, B. L., \& Burch, J. L. (2019). Mechanism of reconnection on kinetic scales based on Magnetospheric Multiscale mission observations. The Astrophysical Journal Letters, 885, L26. https://doi.org/10.3847/2041-8213/ab4b5a

Matteini, L., Alexandrova, O., Chen, C. H. K., \& Lacombe, C. (2017). Electric and magnetic spectra from MHD to electron scales in the magnetosheath. MNRAS, 466, 945-951. https://doi.org/10.1093/mnras/stw3163

Matthaeus, W. H., \& Goldstein, M. L. (1982). Measurement of the rugged invariants of magnetohydrodynamic turbulence in the solar wind. Journal of Geophysical Research, 87, 6011-6028. https://doi.org/10.1029/JA087iA08p06011

Narita, Y., Baumjohann, W., \& Treumann, R. A. (2019). Scaling laws in Hall inertial-range turbulence. Annales Geophysicae, 37, 825-834. https://doi.org/10.5194/angeo-37-825-2019

Parashar, T. N., Chasapis, A., Band yopadhyay, R., Chhiber, R., Matthaeus, W. H., Maruca, B., \& Roytershteyn, V. (2018). Kinetic range spectral features of cross helicity using the Magnetospheric Multiscale spacecraft. Physical Review Letters, 121, 265101. https://doi. org/10.1103/PhysRevLett.121.265101

Pezzi, O., Valentini, F., \& Veltri, P. (2016). Collisional relaxation of fine velocity structures in plasmas. Physical Review Letters, 116, 145001. https://doi.org/10.1103/PhysRevLett.116.145001

Phan, T. D., Eastwood, J. P., Shay, M. A., Drake, J. F., Sonnerup, B. U. Ö., Fujimoto, M., \& Magnes, W. (2018). Electron magnetic reconnection without ion coupling in Earth's turbulent magnetosheath. Nature, 557, 202-206. https://doi.org/10.1038/s41586-018-0091-5

Pollock, C., Moore, T., Jacques, A., Burch, J., Gliese, U., Saito, Y., \& Zeuch, M. (2016). Fast plasma investigation for Magnetospheric Multiscale. Space Science Reviews, 199, 331-406. https://doi.org/10.1007/s11214-016-0245-4

Retinò, A., Sundkvist, D., Vaivads, A., Mozer, F., André, M., \& Owen, C. J. (2007). In situ evidence of magnetic reconnection in turbulent plasma. Nature Physics, 3, 236-238. https://doi.org/10.1038/nphys574

Robert, P., Dunlop, M. W., Roux, A., \& Chanteur, G. (1998). Accuracy of current density determination. ISSI Scientific Reports Series, 1 , 395-418.

Russell, C. T., Anderson, B. J., Baumjohann, W., Bromund, K. R., Dearborn, D., Fischer, D., \& Richter, I. (2016). The Magnetospheric Multiscale magnetometers. Space Science Reviews, 199, 189-256. https://doi.org/10.1007/s11214-014-0057-3

Sahraoui, F., Belmont, G., Pinçon, J., Rezeau, L., Balogh, A., Robert, P., \& Cornilleau-Wehrlin, N. (2004). Magnetic turbulent spectra in the magnetosheath: New insights. Annales Geophysicae, 22, 2283-2288. https://doi.org/10.5194/angeo-22-2283-2004

Sahraoui, F., Goldstein, M. L., Robert, P., \& Khotyaintsev, Y. V. (2009). Evidence of a cascade and dissipation of solar-wind turbulence at the electron gyroscale. Physical Review Letters, 102, 231102. https://doi.org/10.1103/PhysRevLett.102.231102

Saur, J., Politano, H., Pouquet, A., \& Matthaeus, W. H. (2002). Evidence for weak MHD turbulence in the middle magnetosphere of Jupiter Astronomy \& Astrophysics, 386, 699-708. https://doi.org/10.1051/0004-6361:20020305

Shuster, J. R., Gershman, D. J., Chen, L. J., Wang, S., Bessho, N., Dorelli, J. C., \& Viñas, A. F. (2019). MMS measurements of the Vlasov equation: Probing the electron pressure divergence within thin current sheets. Geophysical Research Letters, 46, 7862-7872. https://doi. org/10.1029/2019GL083549

Stawarz, J. E., Eastwood, J. P., Phan, T. D., Gingell, I. L., Shay, M. A., Burch, J. L., \& Franci, L. (2019). Properties of the turbulence associated with electron-only magnetic reconnection in Earth's magnetosheath. The Astrophysical Journal Letters, 877, L37. https://doi. org/10.3847/2041-8213/ab21c8

Stawarz, J. E., Eriksson, S., Wilder, F. D., Ergun, R. E., Schwartz, S. J., Pouquet, A., \& Sturner, A. P. (2016). Observations of turbulence in a Kelvin-Helmholtz event on 8 September 2015 by the Magnetospheric Multiscale mission. Journal of Geophysical Research: Space Physics, 121, 11021-11034. https://doi.org/10.1002/2016JA023458

Sundkvist, D., Retinò, A., Vaivads, A., \& Bale, S. D. (2007). Dissipation in turbulent plasma due to reconnection in thin current sheets. Physical Review Letters, 99, 025004. https://doi.org/10.1103/PhysRevLett.99.025004

Taylor, G. I. (1938). The spectrum of turbulence. Proceedings of the Royal Society of London. Series A, 164, 476-490. https://doi.org/10.1098/ rspa.1938.0032

Torbert, R. B., Burch, J. L., Giles, B. L., Gershman, D., Pollock, C. J., Dorelli, J., \& Bounds, S. (2016). Estimates of terms in Ohm's law during an encounter with an electron diffusion region. Geophysical Research Letters, 43, 5918-5925. https://doi.org/10.1002/2016GL069553

Vörös, Z., Yordanova, E., Varsani, A., Genestreti, K. J., Khotyaintsev, Y. V., Li, W., \& Saito, Y. (2017). MMS observation of magnetic reconnection in the turbulent magnetosheath. Journal of Geophysical Research: Space Physics, 122, 11442-11467. https://doi. org/10.1002/2017JA024535

Webster, J. M., Burch, J. L., Reiff, P. H., Daou, A. G., Genestreti, K. J., Graham, D. B., \& Wilder, F. (2018). Magnetospheric Multiscale dayside reconnection electron diffusion region events. Journal of Geophysical Research: Space Physics, 123, 4858-4878. https://doi. org/10.1029/2018JA025245

Yordanova, E., Vörös, Z., Varsani, A., Graham, D. B., Norgren, C., Khotyaintsev, Y. V., \& Saito, Y. (2016). Electron scale structures and magnetic reconnection signatures in the turbulent magnetosheath. Geophysical Research Letters, 43, 5969-5978. https://doi. org/10.1002/2016GL069191

Zhuravleva, I., Churazov, E., Schekochihin, A. A., Allen, S. W., Arévalo, P., Fabian, A. C., \& Werner, N. (2014). Turbulent heating in galaxy clusters brightest in X-rays. Nature, 515, 85-87. https://doi.org/10.1038/nature13830 